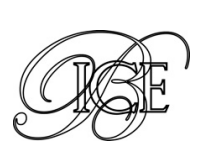

Subdirección General de Comercio Internacional de Material de Defensa y Doble Uso*

\title{
EXPORTACIONES ESPAÑOLAS DE MATERIAL DE DEFENSA, DE OTRO MATERIAL Y DE PRODUCTOS Y TECNOLOGÍAS DE DOBLE USO EN EL PRIMER SEMESTRE DE 2015
}

El informe de estadísticas del primer semestre de 2015 que se publica en este número del Boletín Económico de ICE es enviado a las Comisiones de Asuntos Exteriores y de Defensa del Congreso de los Diputados. De esta manera, se da cumplimiento a lo dispuesto en el artículo 16 de la Ley 53/2007, de 28 de diciembre, sobre el control del comercio exterior de material de defensa y de doble uso.

Palabras clave: armas de caza y deportivas, productos y tecnologías de doble uso, exportaciones autorizadas y realizadas, foros internacionales de control y no proliferación, cláusula catch-all, embargos, denegaciones, Tratado Internacional sobre el Comercio de Armas, proliferación, armas de destrucción masiva.

Clasificación JEL: F10, H56.

\section{Introducción}

Este informe incorpora las exportaciones autorizadas y realizadas de material de defensa, de otro material y de productos y tecnologías de doble uso del primer semestre de 2015 de acuerdo con lo dispuesto en el artículo 16 de la Ley 53/2007, de 28 de diciembre, sobre el control del comercio exterior de material de defensa y de doble uso. De esta manera, se da cumplimiento a la obligación del Gobierno, consistente en presentar semestralmente al Congreso de los Diputados la información relativa a estas operaciones.

El análisis detallado de la política de control de las exportaciones de material de defensa, de otro material y de productos y tecnologías de doble uso

\footnotetext{
* Ministerio de Economía y Competitividad. Este artículo ha sido elaborado por Ramón Muro Martínez. Subdirector General de Comercio Internacional de Material de Defensa y Doble Uso y por José Luis Valenzuela Vadillo, Jefe de Servicio.

Versión de diciembre de 2015.
}

llevada a cabo por la Secretaría de Estado de Comercio y la Junta Interministerial Reguladora del Comercio Exterior de Material de Defensa y de Doble Uso (JIMDDU) se realizará, como viene siendo habitual, con motivo de la publicación de las estadísticas de todo el año 2015.

\section{Material de defensa}

El valor de las exportaciones realizadas en el primer semestre de 2015 fue de 1.727,2 millones de euros, con un aumento del 25,3 por 100 frente a lo obtenido en el primer semestre del año 2014 (1.378,5 millones de euros).

Las exportaciones realizadas (1.727,2 millones de euros) representaron un 122,5 por 100 de lo autorizado (1.409,4 millones de euros) en el primer semestre de 2015.

En lo referente al desglose por países de los productos exportados, las exportaciones realizadas a $\triangle$ 
los países de la UE supusieron el 31,3 por 100 del total (539,5 millones de euros). La mayor parte del comercio tuvo lugar con aquellos países de la Unión Europea -Reino Unido, Francia, Alemania e Italiacon los que España mantiene proyectos industriales militares en común, como son concretamente los programas del avión de combate (EF 2000) y del avión de transporte militar (A400M) europeos. Es preciso resaltar que las exportaciones realizadas, en conjunto, a estos cuatro países ascendieron a 509,4 millones de euros (29,5 por 100 del total). El importe fue de 226,7 millones de euros al Reino Unido (un avión de transporte, repuestos de aviones de reabastecimiento en vuelo, partes y componentes en el marco de los dos programas de cooperación de aeronaves antes citados, componentes y cargas para misil, partes, piezas y componentes de helicópteros, componentes y datos técnicos relativos a una programa de construcción de vehículos blindados, pólvora, munición y componentes de artillería y rifles de caza), 158,0 millones de euros a Francia (un avión de transporte militar, partes y componentes para el avión de transporte militar europeo, componentes para helicópteros, partes y piezas para misiles, equipos para un programa de comunicaciones electrónicas, material de transmisiones y circuitos electrónicos, combustible con especificaciones militares, pólvora, componentes de munición de artillería, gafas de visión nocturna, pistolas de tiro deportivo y rifles de caza con componentes), 87,2 millones de euros a Alemania (partes y componentes para los programas de cooperación de aeronaves, componentes de helicóptero, equipos para programas de misiles, equipos de un programa de comunicaciones electrónicas, sistemas de guerra electrónica, una estación de comunicación vía satélite, circuitos electrónicos, partes para vehículo blindado, un contenedor, pólvora, componentes de munición de artillería, pistolas de uso deportivo y rifles de caza con componentes) y 37,5 millones de euros a Italia (partes y componentes para los programas de cooperación de aeronaves y otras aeronaves, componentes de helicóptero, equipos de un programa de comunicaciones electrónicas, equipos de cifrado, detonador eléctrico, componentes de equipos de guerra electrónica, combustible con especificaciones militares, componentes de artillería naval, componentes de hélices de buques de guerra, armas ligeras, subconjuntos de cañón antiaéreo, partes y piezas para vehículos blindados, munición de artillería, pólvora, pistolas de tiro deportivo y rifles de caza con componentes).

El 33,7 por 100 de las exportaciones españolas tuvo como destino a países OTAN $(581,6$ millones de euros). Si se suman las exportaciones a países de la UE y la OTAN, el valor de lo exportado fue de 584,4 millones de euros y un 33,9 por 100 . El resto de las exportaciones (1.142,8 millones de euros, 66,1 por 100), una vez descontadas todas aquellas con destino a países de la UE y de la OTAN, se repartieron entre 46 países, entre los que destacaron Arabia Saudí con 447,6 millones de euros en dos aviones de reabastecimiento en vuelo, repuestos para aviones de transporte, partes, piezas y reparación de motores de cazas de combate, munición ligera enviada sin cobro del valor al tratarse de material de reposición, una estación de control remoto para armas y pistolas de uso deportivo para una $\triangleright$

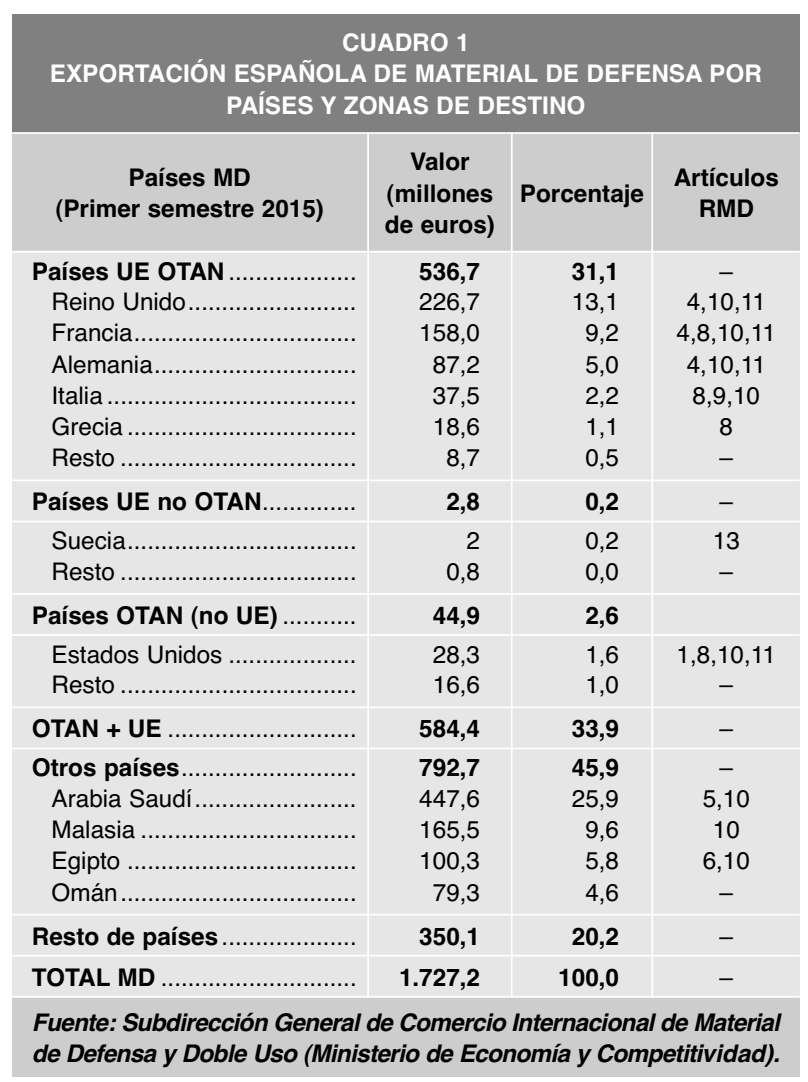


empresa particular; Malasia con 165,5 millones de euros en un avión de transporte y visores nocturnos con accesorios para las FFAA; Egipto con 100,3 millones de euros en cuatro aviones de transporte, repuestos, componentes y motores para diversas aeronaves, piezas de repuestos y soporte técnico para motores de corbetas de fabricación española, recambios para motores de diversos vehículos terrestres y recambios de maquinaria para la fabricación de cadenas de vehículos blindados, y Omán con 79,3 millones de euros en un avión de transporte.

Del análisis de lo exportado por categorías de productos, la de «aeronaves» (1.497,5 millones de euros, 86,7 por 100) fue la partida más importante.

Hubo tres denegaciones de material de defensa en el primer semestre de 2015 consistentes en dos licencias individuales con destino a Rusia de un rifle de caza y sesenta pistolas deportivas y una licencia individual a Venezuela de recambios de motor de camión de transporte y herramientas para reparación de vehículo blindado de ruedas. Las dos primeras licencias fueron denegadas en aplicación del artículo 7.1, epígrafe e) del Real Decreto 679/2014, de 1 de agosto, por el que se aprueba el Reglamento de control del comercio exterior de material de defensa, de otro material y de productos y tecnologías de doble uso, y del criterio 1 de la Posición Común 2008/944/PESC, de 8 de diciembre, por la que se definen las normas comunes que rigen el control de las exportaciones de tecnología y equipos militares, dada la existencia de un embargo de la Unión Europea a dicho país de 31 de julio y 8 de septiembre de 2014. La tercera licencia fue denegada en aplicación de los epígrafes a), b), c) y d) del mismo artículo, así como de los criterios 2 (respeto de derechos humanos) y 3 (situación interna) de la citada Posición Común.

\section{Otro material}

Por lo que respecta a las operaciones realizadas de material policial y de seguridad, lo exportado ascendió a 1,2 millones de euros frente a 6,3 millones de euros en el mismo período de 2014, con una disminución del 81,4 por 100. Las exportaciones realizadas (1,2 millones de euros) supusieron un 426,5 por 100 de lo autorizado en el mismo período (274.575 euros), al efectuarse envíos con cargo a licencias autorizadas en 2014 y en vigor en el primer semestre de 2015.

Los destinos fueron: Perú (950.849 euros, 81,2 por 100), Túnez (207.200 euros, 17,7 por 100), Portugal (13.015 euros, 1,1 por 100) y Togo (una donación por 0 euros, 0 por 100).

En lo relativo a las operaciones de armas de caza y deportivas (escopetas de caza, cartuchos y sus piezas y recambios), las exportaciones realizadas ascendieron a 15,0 millones de euros, un 13,6 por 100 de aumento en relación con el mismo periodo de 2014 (13,2 millones de euros). Las exportaciones realizadas (15,0 millones de euros) representaron un 17,3 por 100 de lo autorizado (86,9 millones de euros).

El destino principal fue Estados Unidos con 1,9 millones de euros y un 12,7 por 100. Las restantes exportaciones se repartieron entre 48 países.

El 83,5 por 100 de las exportaciones correspondieron a cartuchos de caza, un 10,6 por 100 a escopetas, un 5,9 por 100 a vainas, perdigones y pólvora y el 0,02 por 100 restante a componentes de armas de caza.

No hubo denegaciones de arma de caza en el primer semestre de 2015.

\section{Productos y tecnologías de doble uso}

En lo concerniente a los productos y tecnologías de doble uso, el valor de las exportaciones españolas realizadas en el primer semestre de 2015 totalizó 81,2 millones de euros, con un incremento del 29,1 por 100 en relación a lo exportado en el primer semestre del año anterior (62,9 millones de euros). Las exportaciones realizadas $(81,2$ millones de euros) supusieron un 44,7 por 100 de lo autorizado en el mismo período (181,8 millones de euros).

Los dos principales destinos de las exportaciones españolas fueron Estados Unidos, con 16,5 $\triangleright$ 
millones de euros y un 20,3 por 100, y la RP China, con 12,3 millones de euros y un 15,1 por 100. Las exportaciones restantes correspondieron a 50 países.

Las exportaciones a Estados Unidos se materializaron en electrodos de grafito para la siderurgia, sustancias químicas para el sector industrial, válvulas industriales para el tratamiento de aguas, una cámara de infrarrojos para el sector industrial, controles numéricos y un centro de mecanizado para el sector industrial, máquinas fresadoras, de encintado y mecanizado destinadas a la industria aeronáutica, y diverso material electrónico y tarjetas para el sector de telecomunicaciones.

Los envíos a la RP China se concretaron en un equipo de inspección para una central nuclear de uso civil, sustancias químicas para uso en laboratorio, un sistema de inspección por ultrasonidos de piezas en materiales compuestos para el sector aeronáutico, componentes internos de columnas en el sector petroquímico, condensadores eléctricos fijos para los sectores de generación de energía eléctrica y de transportes, circuitos integrados y memorias destinados a satélites y al sector de telecomunicaciones, detectores lineales para los sectores de transportes y de control medioambiental, sistemas de control para UAV en el sector aeronáutico, máquinas de fresado para los sectores del petróleo y automoción, válvulas en acero inoxidable para el sector petroquímico, centros de mecanizado para el sector de automoción y circuitos integrados para el sector de telecomunicaciones.

Las exportaciones a Irán sufrieron un descenso del 40,4 por 100 con 5,9 millones de euros respecto al primer semestre de 2014 (9,9 millones de euros). Los envíos consistieron en válvulas de control en acero inoxidable para los sectores del gas y petroquímico, así como conjuntos para hornos industriales en el sector siderúrgico.

Estas operaciones fueron analizadas teniendo en cuenta las resoluciones de Naciones Unidas, además de las decisiones y los reglamentos de la Unión Europea aprobados desde diciembre de 2006 sobre medidas restrictivas contra Irán.
Todas las licencias aprobadas con destino a Irán fueron el resultado de la aplicación de la cláusula catch-all de acuerdo con el artículo 4 del Reglamento (CE) $n^{\circ} 428 / 2009$ del Consejo, de 5 de mayo de 2009, por el que se establece un régimen comunitario de control de las exportaciones, la transferencia, el corretaje y el tránsito de productos y tecnologías de doble uso, y sucesivas modificaciones.

El empleo de la citada cláusula dio lugar a un considerable incremento de las licencias de exportación, habiéndose aprobado exclusivamente aquellas que no implicaban riesgo alguno de desvío hacia un uso militar o utilización en programas de fabricación de armas de destrucción masiva.

La cláusula catch-all es aplicable a exportaciones de productos no incluidos en las listas de control cuando exista un posible riesgo de desvío a programas de fabricación de armas de destrucción masiva o cuando el país de destino esté sometido a un embargo de armas y los productos vayan a tener un uso final militar. Su empleo permite estudiar detalladamente tales envíos.

Por categorías de productos destacó, como grupo prioritario de las exportaciones, la de «tratamiento de los materiales» con 52,2 millones de euros y un 64,3 por 100 .

Durante el primer semestre de 2015 fueron informadas negativamente tres licencias individuales de exportación. La primera tuvo como destino a Pakistán y consistió en 100 Tn de sulfuro de sodio. Se denegó por no ofrecer las suficientes garantías el usuario final. La segunda fue a Irán de componentes de válvulas, por posible riesgo de desvío al programa nuclear, y la tercera a Myanmar de dos máquinas-herramienta, por la existencia de un embargo de la UE de 28 de octubre de 1996. Fueron renovadas una denegación en el Grupo Australia, cuatro en el Grupo de Suministradores Nucleares y tres en el Régimen de Control de la Tecnología de Misiles.

Por último, cabe decir que la cláusula catch-all fue aplicada en un caso, consistente en la posible exportación a Irán de repuestos de válvulas de seguridad. 


\section{Anexo estadístico}

\begin{tabular}{|c|c|c|c|c|c|}
\hline \multicolumn{6}{|c|}{$\begin{array}{l}\text { 1. EXPORTACIONES AUTORIZADAS DE MATERIAL DE DEFENSA POR PAÍS Y NÚMERO DE LICENCIAS } \\
\text { PRIMER SEMESTRE } 2015\end{array}$} \\
\hline País & $\mathbf{N}^{\circ}$ Licencias & Valor euros & País & $\mathrm{N}^{\circ}$ Licencias & Valor euros \\
\hline 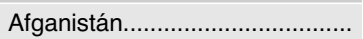 & 2 & 150.000 & Italia .......... & 57 & 64.733 .815 \\
\hline 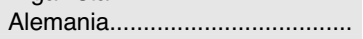 & 77 & 149.885 .009 & Japón............................... & 1 & 0 \\
\hline 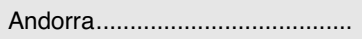 & 13 & 65.281 & 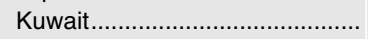 & 1 & 0 \\
\hline 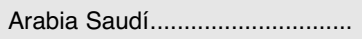 & 8 & 27.113.193 & 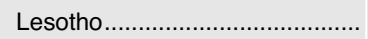 & 1 & 30.000 .000 \\
\hline Argelia & 4 & 120.000 & Lituania ................................. & 1 & 400.000 \\
\hline 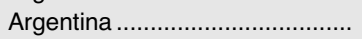 & 20 & 6.175 .060 & Luxemburgo............................. & 4 & 127.237 \\
\hline 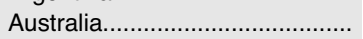 & 8 & 22.068 .693 & 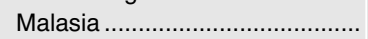 & 3 & 200.000 \\
\hline Austria & 9 & 480.000 & 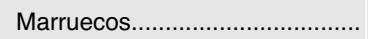 & 9 & 38.866 .600 \\
\hline 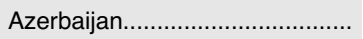 & 1 & 71.160 .782 & México ….............................. & 2 & 80.000 .000 \\
\hline 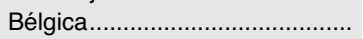 & 13 & 6.180 .443 & Noruega.............................. & 12 & 2.630 .113 \\
\hline 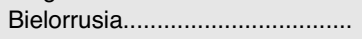 & 2 & 0 & Nueva Caledonia (Francia) ....... & 1 & 15.000 \\
\hline 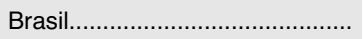 & 15 & 5.377 .656 & 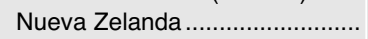 & 2 & 5.741 .250 \\
\hline 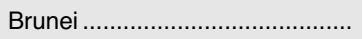 & 1 & 10.000 .000 & 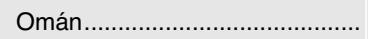 & 3 & 1.561 .800 \\
\hline 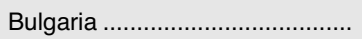 & 2 & 0 & Países Bajos ............................ & 5 & 255.814 \\
\hline 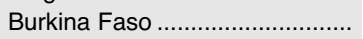 & 1 & 0 & 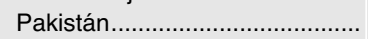 & 3 & 30.504 .000 \\
\hline 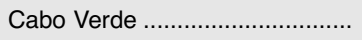 & 1 & 0 & Paraguay & 1 & 0 \\
\hline 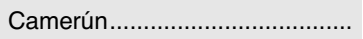 & 1 & 30.000 .000 & 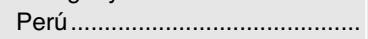 & 9 & 3.339 .126 \\
\hline 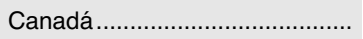 & 33 & 4.505 .450 & 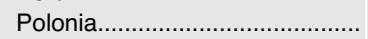 & 3 & 1.691 .548 \\
\hline 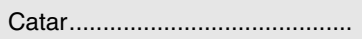 & 1 & 0 & 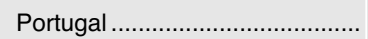 & 21 & 2.944.819 \\
\hline 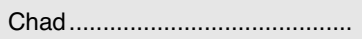 & 1 & 0 & Reino Unido............................ & 61 & 202.483 .904 \\
\hline Chile & 12 & 2.766 .850 & 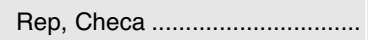 & 6 & 0 \\
\hline 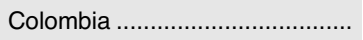 & 2 & 2.178 .454 & 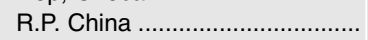 & 1 & 250.000 \\
\hline 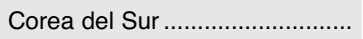 & 1 & 0 & 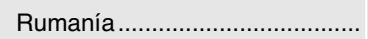 & 1 & 12.600 \\
\hline Croacia & 1 & 0 & Rusia & 2 & 2.200 .000 \\
\hline 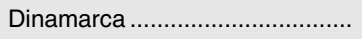 & 9 & 1.089 .447 & 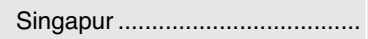 & 2 & 198.000 \\
\hline 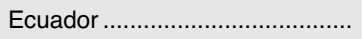 & 1 & 69.631 .271 & 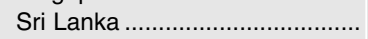 & 1 & 10.000 \\
\hline 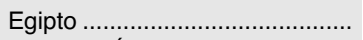 & 16 & 1.960 .057 & Sudáfrica ............................ & 3 & 2.500 .000 \\
\hline Emiratos Árabes Unidos.............. & 6 & 0 & Suecia................................ & 9 & 2.026 .489 \\
\hline 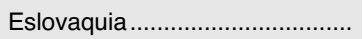 & 1 & 34.960 & 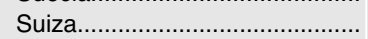 & 8 & 400.000 \\
\hline 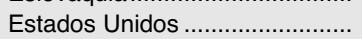 & 174 & 131.393 .269 & 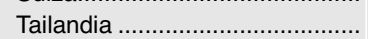 & 9 & 2.633 .470 \\
\hline 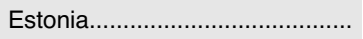 & 2 & 15.000 & 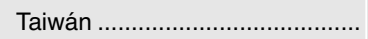 & 1 & 6.000 .000 \\
\hline 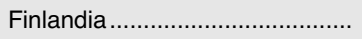 & 3 & 300.000 & 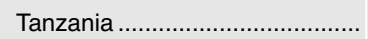 & 3 & 2.470 \\
\hline 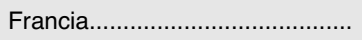 & 56 & 20.346 .872 & 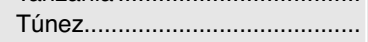 & 3 & 9.302 .572 \\
\hline Grecia & 3 & 15.000 & 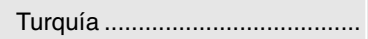 & 10 & 32.079 .350 \\
\hline Groenlandia (Dinamarca) ........... & 1 & 14.213 .000 & 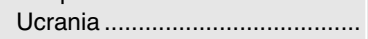 & 1 & 9.290 \\
\hline 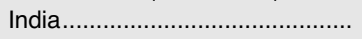 & 14 & 8.111 .251 & 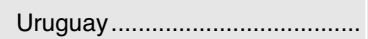 & 3 & 42.040 \\
\hline 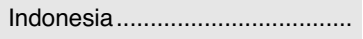 & 13 & 7.523 .775 & 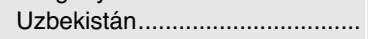 & 1 & 180.000 .000 \\
\hline Irak & 1 & 17.865 .000 & 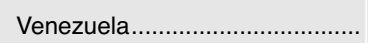 & 1 & 92.500 .000 \\
\hline Irlanda.............................................. & 1 & 1.311 .586 & TOTAL & 806 & 1.409 .385 .816 \\
\hline 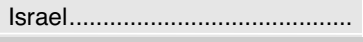 & 11 & 1.687 .150 & IUIAL. & 806 & 1.409 .385 .816 \\
\hline
\end{tabular}




\section{Subdirección General de Comercio Internacional de Material de Defensa y Doble Uso}

\begin{tabular}{|c|c|c|c|c|c|c|c|c|c|c|c|}
\hline 2. EXPORTAC & CIONES A & UTORIZAI & DAS DE M & $\begin{array}{l}\text { IATERIAL L } \\
\text { PRIMER S }\end{array}$ & $\begin{array}{l}\text { DEFENS: } \\
\text { IESTRE ? }\end{array}$ & $\begin{array}{l}\text { POR Pk } \\
015\end{array}$ & $\mathbf{Y}$ & CULOS & PROL & ICTOS & \\
\hline \multirow{2}{*}{ País } & \multicolumn{11}{|c|}{ Artículos y valor en euros } \\
\hline & 1 & 2 & 3 & 4 & 5 & 6 & 7 & 8 & 9 & 10 & 11 \\
\hline \multicolumn{12}{|l|}{ Afganistán............................... } \\
\hline Alemania & 334.483 & & 782 & 27.056 .013 & 0 & 42.228 & & 0 & & 103.571 .152 & 18.880 .351 \\
\hline 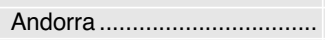 & 46.494 & & 18.787 & & & & & & & & \\
\hline Arabia Saudí ........................... & & & 6.925 .020 & 17.343 .173 & 645.000 & & & & & 2.200 .000 & \\
\hline 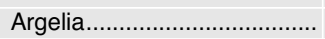 & & & & & & & & & 120.000 & 0 & \\
\hline 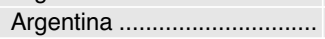 & 19.910 & & 2.210 .150 & 2.145 .000 & & & & & & & 1.800 .000 \\
\hline 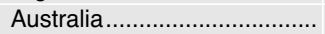 & 500.000 & 24.500 & & 0 & & & & & 1.544 .193 & 0 & 20.000 .000 \\
\hline Austria.............................. & 480.000 & & & & & 0 & & & & 0 & \\
\hline \multicolumn{12}{|l|}{ Azerbaiján............................. } \\
\hline Bélgica ................................... & 430.000 & & 2.924 .143 & & & 2.826 .300 & & & & 0 & \\
\hline Bielorrusia ............................. & & & & & & & 0 & & & & \\
\hline 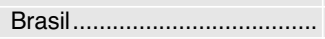 & 3.542 & & 612.556 & & & & & & 1.736 .091 & 1.300 .000 & 1.725 .467 \\
\hline Brunei & & & & & & & & & & 10.000 .000 & \\
\hline 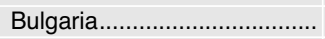 & & & & & 0 & & & & & 0 & \\
\hline 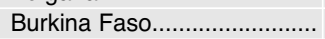 & & & & & & & & & & 0 & \\
\hline 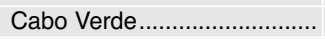 & & & & & & & & & & 0 & \\
\hline 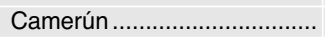 & & & & & & & & & & 30.000 .000 & \\
\hline 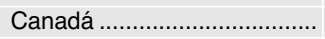 & & & & 847 & & 2.642 .044 & & & 1.862 .559 & 0 & 0 \\
\hline \multicolumn{12}{|l|}{ Catar …………… } \\
\hline Chad .................................. & & & & & & & & & & 0 & \\
\hline 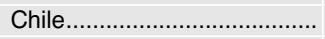 & 25.030 & & 212.225 & & & 2.529 .595 & & & & 0 & \\
\hline 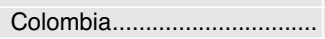 & & & 2.178 .454 & & & & & & & & \\
\hline Corea del Sur .......................... & & & & & & & & & & 0 & \\
\hline 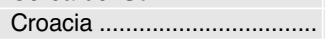 & & & & & & & & & & 0 & \\
\hline Dinamarca ............................... & 25.000 & 0 & 0 & & & & & 980.000 & & 84.447 & \\
\hline \multicolumn{12}{|l|}{ 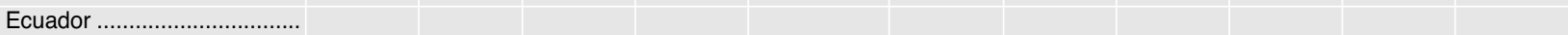 } \\
\hline Egipto.......................................... & & & & & 0 & 689.102 & & & 1.180 .850 & 90.105 & 0 \\
\hline Emiratos Árabes Unidos........ & & 0 & 0 & 0 & & & & & & 0 & \\
\hline 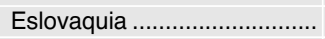 & 34.960 & & & & & & & & & & \\
\hline Estados Unidos.......................... & 4.194 .454 & & 1.619 .941 & 5.251 .861 & & 14.996 & & 77.727 .000 & 0 & 6.028 .733 & 32.398 .094 \\
\hline 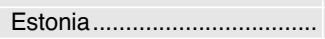 & 15.000 & & & & & & & & & 0 & \\
\hline 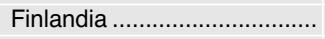 & 300.000 & & & & & & & & & 0 & \\
\hline 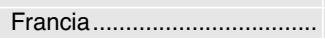 & 1.002 .600 & & 180.818 & 2.042 .044 & & & & 10.853 .300 & 84.330 & 1.739 .439 & 2.291 .840 \\
\hline Grecia ........................... & 15.000 & & & & & & & & & 0 & \\
\hline Groenlandia (Dinamarca) ...... & & & & & & & & 14.213 .000 & & & \\
\hline 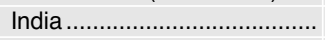 & & & & & & 6.527 & & & 446.777 & 532.974 & 7.124 .973 \\
\hline Indonesia ............................ & & 784.862 & 1.355 .980 & & & 3.919 .825 & & & & 1.000 .000 & \\
\hline Irak & & & 17.865 .000 & & & & & & & & \\
\hline Irlanda & & & & & 1.311 .586 & & & & & & \\
\hline 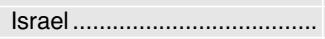 & & 0 & & 219.950 & 0 & & & & & 0 & 0 \\
\hline 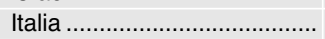 & 47.000 & 6.250 .520 & 5.769 .739 & & & 2.400 .000 & & 9.000 & 2.301 .888 & 46.039 .058 & 1.916 .610 \\
\hline 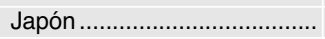 & & & & & & & & & & 0 & \\
\hline 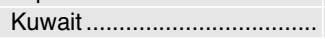 & & & 0 & & & & & & & & \\
\hline Lesotho ............................ & & & & & & & & & & 30.000 .000 & \\
\hline Lituania ............................. & 400.000 & & & & & & & & & & \\
\hline Luxemburgo........................... & & & & 0 & & & & & & 125.255 & \\
\hline Malasia .................................. & & & & & & & & & & 200.000 & \\
\hline Marruecos............................ & & & 8.029 .000 & 290.700 & & & & 46.900 & & 30.000 .000 & 500.000 \\
\hline México …….......................... & & & & & & & & & & 80.000 .000 & \\
\hline 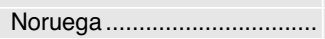 & 1.100 .000 & & 382.050 & 919.000 & & 23.063 & & 206.000 & & 0 & 0 \\
\hline Nueva Caledonia (Francia)... & 15.000 & & & & & & & & & & \\
\hline 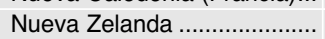 & 1.100 .000 & & 4.641 .250 & & & & & & & & \\
\hline Omán & & & 1.318 .800 & 243.000 & & & & & & & \\
\hline 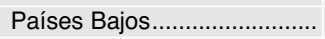 & & & & & & 0 & & & 255.814 & 0 & \\
\hline 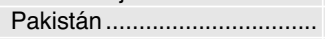 & & & & & & 504.000 & & & & 30.000 .000 & \\
\hline 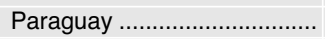 & 0 & & & & & & & & & & \\
\hline Perú & & 1.310 .640 & 12.847 & & 384.443 & 116.716 & & & & 14.480 & 1.500 .000 \\
\hline Polonia & & & 1.691 .548 & & & & & & & & \\
\hline Portugal ............................ & 1.437 .057 & & 450.000 & 908.017 & & 0 & & 121.995 & & & 27.750 \\
\hline Reino Unido ............................ & 1.695 & & 1.490 .678 & 32.492 .467 & & 102.427 & & 0 & & 168.396 .637 & 0 \\
\hline Rep. Checa.......................... & & 0 & & & & 0 & & & & 0 & \\
\hline R.P. China ............................. & 250.000 & & & & & & & & & & \\
\hline Rumanía ............................. & 12.600 & & & & & & & & & & \\
\hline Rusia.......................................... & 2.200 .000 & & & & & & & & & & \\
\hline Singapur ................................... & & & & & & & & & & 0 & \\
\hline
\end{tabular}


EXPORTACIONES ESPAÑOLAS DE MATERIAL DE DEFENSA...

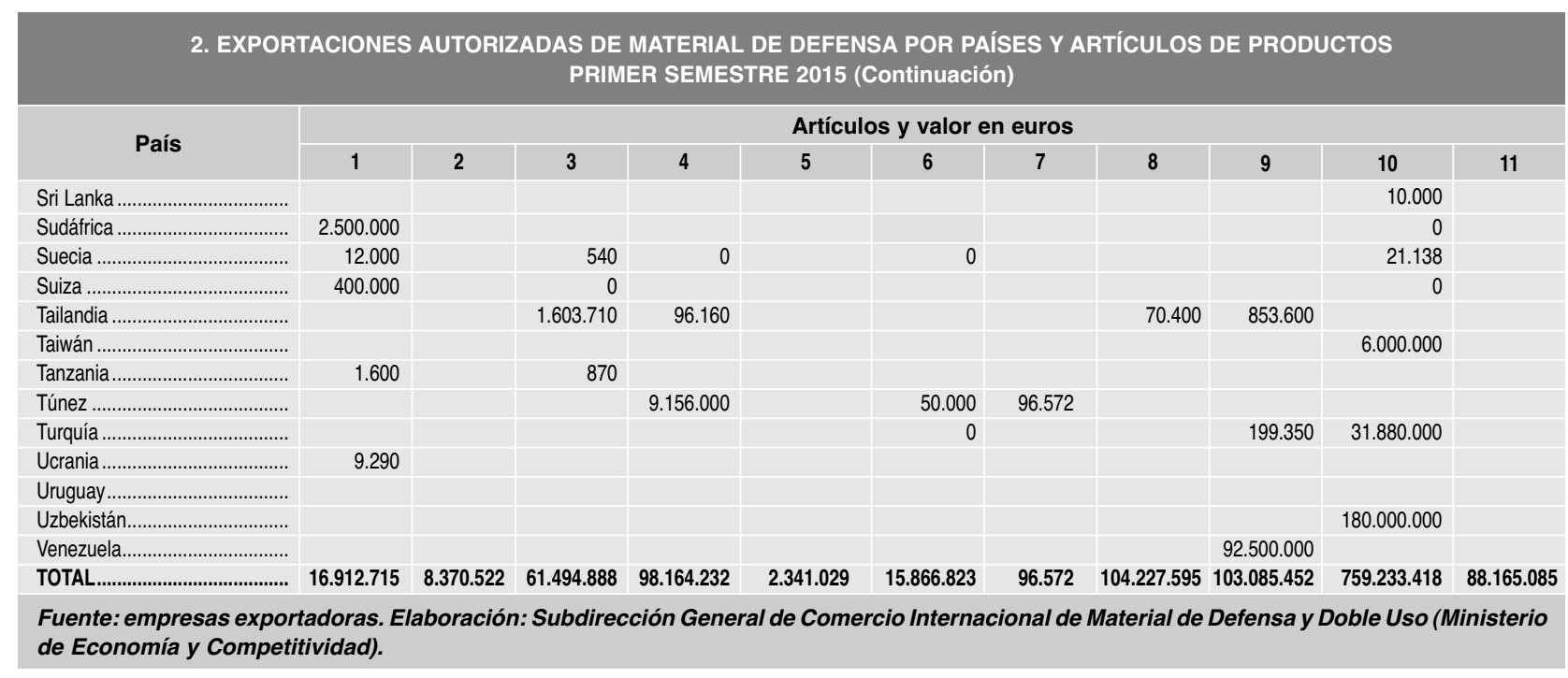




\section{Subdirección General de Comercio Internacional de Material de Defensa y Doble Uso}

2. EXPORTACIONES AUTORIZADAS DE MATERIAL DE DEFENSA POR PAÍSES Y ARTÍCULOS DE PRODUCTOS PRIMER SEMESTRE 2015 (Continuación)

\begin{tabular}{|c|c|c|c|c|c|c|c|c|c|c|c|c|}
\hline \multirow{2}{*}{ País } & \multicolumn{11}{|c|}{ Artículo y valor en euros } & \multirow{2}{*}{$\begin{array}{l}\text { Total valor } \\
\text { euros }\end{array}$} \\
\hline & 12 & 13 & 14 & 15 & 16 & 17 & 18 & 19 & 20 & 21 & 22 & \\
\hline 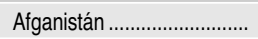 & & & & 150.000 & & & & & & & & 150.000 \\
\hline 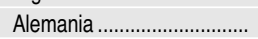 & & & & 0 & & & & & & & & 149.885 .009 \\
\hline 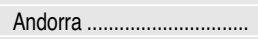 & & & & & & & & & & & & 65.281 \\
\hline Arabia Saudí ......................... & & & & & & & & & & & & 27.113 .193 \\
\hline 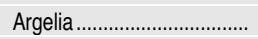 & & & & 0 & & & & & & & & 120.000 \\
\hline 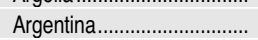 & & & & 0 & & & & & & & & 6.175 .060 \\
\hline Australia ................................ & & & & & & & & & & & & 22.068 .693 \\
\hline Austria & & & & & & & & & & & & 480.000 \\
\hline Azerbaiján ........................... & & & & 71.160 .782 & & & & & & & & 71.160 .782 \\
\hline 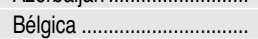 & & & & & & & & & & & & 6.180 .443 \\
\hline 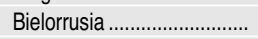 & & & & & & & & & & & & 0 \\
\hline 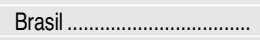 & & & & & & & & & & & & 5.377 .656 \\
\hline 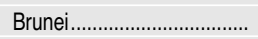 & & & & & & & & & & & & 10.000 .000 \\
\hline 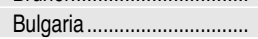 & & & & & & & & & & & & 0 \\
\hline Burkina Faso ....................... & & & & & & & & & & & & 0 \\
\hline Cabo Verde .............................. & & & & & & & & & & & & 0 \\
\hline 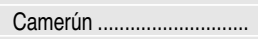 & & & & & & & & & & & & 30.000 .000 \\
\hline 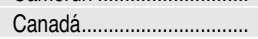 & & & & & & & & & & & 0 & 4.505 .450 \\
\hline 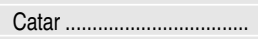 & & & 0 & & & & & & & & & 0 \\
\hline 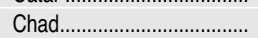 & & & & & & & & & & & & 0 \\
\hline 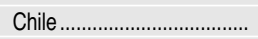 & & & & 0 & & & & & & & & 2.766 .850 \\
\hline 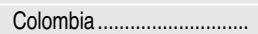 & & & & & & & & & & & & 2.178 .454 \\
\hline Corea del Sur .......................... & & & & & & & & & & & & 0 \\
\hline 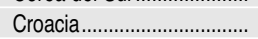 & & & & & & & & & & & & 0 \\
\hline 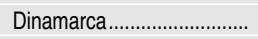 & & & & & & & & & & & & 1.089 .447 \\
\hline 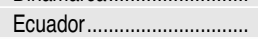 & & & & 69.631 .271 & & & & & & & & 69.631 .271 \\
\hline Egipto & & & & 0 & & & & & & & & 1.960 .057 \\
\hline Emiratos Árabes Unidos .... & & & & 0 & & & & & & & & 0 \\
\hline Eslovaquia.............................. & & & & & & & & & & & & 34.960 \\
\hline 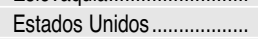 & & & 4.158 .190 & 0 & & & & & & & & 131.393 .269 \\
\hline 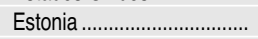 & & & & & & & & & & & & 15.000 \\
\hline 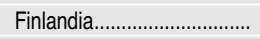 & & & & & & & & & & & & 300.000 \\
\hline 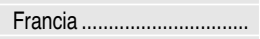 & & & & 2.152 .501 & & & & & & & & 20.346 .872 \\
\hline Grecia......................................... & & & & & & & & & & & 0 & 15.000 \\
\hline Groenlandia (Dinamarca)... & & & & & & & & & & & & 14.213 .000 \\
\hline India ........................................ & & & & & & & & & & & & 8.111 .251 \\
\hline 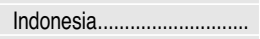 & & & & 463.108 & & & & & & & & 7.523 .775 \\
\hline Irak & & & & & & & & & & & & 17.865 .000 \\
\hline Irlanda & & & & & & & & & & & & 1.311 .586 \\
\hline 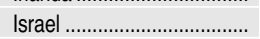 & & & & 1.467 .200 & & & & & & & & 1.687 .150 \\
\hline Italia........................................... & & & & & & & & & & & & 64.733 .815 \\
\hline Japón ......................................... & & & & & & & & & & & & 0 \\
\hline Kuwait ................................ & & & & & & & & & & & & 0 \\
\hline Lesotho ................................ & & & & & & & & & & & & 30.000 .000 \\
\hline Lituania.................................. & & & & & & & & & & & & 400.000 \\
\hline Luxemburgo ............................. & & & & 1.982 & & & & & & & & 127.237 \\
\hline Malasia..................................... & & & & 0 & & & & & & & & 200.000 \\
\hline Marruecos ................................ & & & & 0 & & & & & & & & 38.866 .600 \\
\hline México & & & & & & & & & & & & 80.000 .000 \\
\hline Noruega ...................................... & & & & & & & & & & & & 2.630 .113 \\
\hline Nueva Caledonia (Francia) & & & & & & & & & & & & 15.000 \\
\hline Nueva Zelanda..................... & & & & & & & & & & & & 5.741 .250 \\
\hline Omán & & & & & & & & & & & & 1.561 .800 \\
\hline 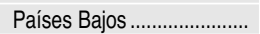 & & & & & & & & & & & & 255.814 \\
\hline Pakistán ............................... & & & & & & & & & & & & 30.504 .000 \\
\hline 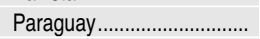 & & & & & & & & & & & & 0 \\
\hline Perú...………………… & & & & & & & & & & & & 3.339 .126 \\
\hline Polonia ........................................ & & & & & & & & & & & & 1.691 .548 \\
\hline 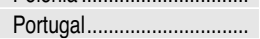 & & & & & & & & & & & & 2.944 .819 \\
\hline Reino Unido ......................... & & & & 0 & & & & & & & & 202.483 .904 \\
\hline Rep. Checa & & & & & & & & & & & & 0 \\
\hline R.P. China .............................. & & & & & & & & & & & & 250.000 \\
\hline 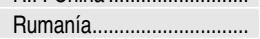 & & & & & & & & & & & & 12.600 \\
\hline 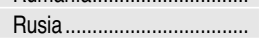 & & & & & & & & & & & & 2.200 .000 \\
\hline Singapur............................... & & & & 198.000 & & & & & & & & 198.000 \\
\hline
\end{tabular}


EXPORTACIONES ESPAÑOLAS DE MATERIAL DE DEFENSA...

\begin{tabular}{|c|c|c|c|c|c|c|c|c|c|c|c|c|}
\hline \multicolumn{13}{|c|}{$\begin{array}{l}\text { 2. EXPORTACIONES AUTORIZADAS DE MATERIAL DE DEFENSA POR PAíSES Y ARTíCULOS DE PRODUCTOS } \\
\text { PRIMER SEMESTRE } 2015 \text { (Continuación) }\end{array}$} \\
\hline \multirow{2}{*}{ País } & \multicolumn{11}{|c|}{ Artículo y valor en euros } & \multirow{2}{*}{$\begin{array}{l}\text { Total valor } \\
\text { euros }\end{array}$} \\
\hline & 12 & 13 & 14 & 15 & 16 & 17 & 18 & 19 & 20 & 21 & 22 & \\
\hline Sri Lanka............................... & & & & & & & & & & & & 10.000 \\
\hline Sudáfrica.............................. & & & & & & & & & & & & 2.500 .000 \\
\hline 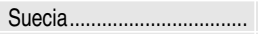 & & 1.992 .811 & & & & & & & & & & 2.026 .489 \\
\hline Suiza...................................... & & & & 0 & & & & & & & & 400.000 \\
\hline 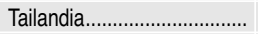 & & & & 9.600 & & & & & & & & 2.633 .470 \\
\hline Taiwán................................... & & & & & & & & & & & & 6.000 .000 \\
\hline Tanzania ............................... & & & & & & & & & & & & 2.470 \\
\hline Túnez.................................. & & & & & & & & & & & & 9.302 .572 \\
\hline Turquía.................................... & & & & & & & & & & & & 32.079 .350 \\
\hline 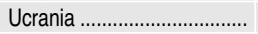 & & & & & & & & & & & & 9.290 \\
\hline Uruguay ……………….......... & & & & 42.040 & & & & & & & & 42.040 \\
\hline Uzbekistán ........................... & & & & & & & & & & & & 180.000 .000 \\
\hline Venezuela ............................. & & & & & & & & & & & & 92.500 .000 \\
\hline TOTAL ………............................ & & 1.992 .811 & 4.158 .190 & 145.276 .484 & & & & & & & 0 & 1.409 .385 .816 \\
\hline
\end{tabular}




\section{Subdirección General de Comercio Internacional de Material de Defensa y Doble Uso}

\begin{tabular}{|c|c|c|c|c|c|c|c|c|c|c|c|}
\hline \multicolumn{12}{|c|}{$\begin{array}{l}\text { 3. EXPORTACIONES REALIZADAS DE MATERIAL DE DEF } \\
\text { PRIMER SEMESTRE } 2015\end{array}$} \\
\hline \multirow{2}{*}{ País } & \multicolumn{11}{|c|}{ Artículo y valor en euros } \\
\hline & 1 & 2 & 3 & 4 & 5 & 6 & 7 & 8 & 9 & 10 & 11 \\
\hline \multicolumn{12}{|l|}{ 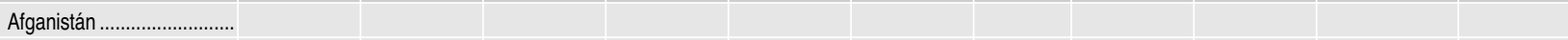 } \\
\hline Alemania............................ & 127.290 & & & 3.468 .612 & & 221.988 & & 0 & & 79.394 .698 & 3.575 .016 \\
\hline Andorra & 14.078 & & 18.364 & & & & & & & & \\
\hline Arabia Saudí........................ & 2.140 & & 0 & & 220.000 & & & & & 447.395 .749 & \\
\hline Argentina ............................. & 19.910 & & 2.460 & & & & & & & & 1.000 .000 \\
\hline 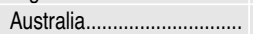 & 8.770 & 14.000 & & 155.500 & & & & & 2.879 .068 & 51.280 .169 & \\
\hline 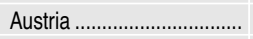 & 48.400 & & & & & & & & & 165.258 & \\
\hline Bahrein ............................... & & & & & & 3.026 .560 & & & & & \\
\hline Bélgica................................. & 26.540 & & 2.908 .320 & & & 250.000 & & & & & \\
\hline Brasil.............................. & 10.297 & & & 612.556 & & & & & & 10.989 .338 & 244.372 \\
\hline Brunei ................................ & & & & & & & & & & 86.597 & \\
\hline 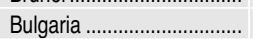 & & & & & & & & 84.679 & & & \\
\hline Burkina Faso & 578 & & & & & & & & & & \\
\hline \multicolumn{12}{|l|}{ Camerún ............................. } \\
\hline Canadá................................ & & & & 1.068 & & 610.460 & & & & 86.719 & 270 \\
\hline 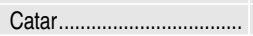 & & & & & & 276.990 & & & & & \\
\hline Chile ..................................... & 7.045 & & 1.166 .972 & & & 324.257 & & & & 673.591 & \\
\hline Colombia ............................... & & & 0 & 2.178 .454 & & & & & & 3.641 .634 & \\
\hline Corea del Sur ....................... & & & & & & & & 10.500 & & 2.784 .559 & 1.949 .824 \\
\hline Dinamarca ........................... & & 0 & 977.280 & 0 & & & & 0 & & & \\
\hline 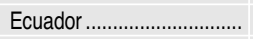 & & & & & & & & & & 1.668 .481 & \\
\hline Egipto …………………. & & & & & & 1.097 .651 & & & 894.788 & 98.342 .826 & \\
\hline Emiratos Árabes Unidos.... & & & & & & & & & & 1.240 .853 & \\
\hline 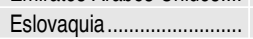 & 5.520 & & & & & & & & & & \\
\hline Eslovenia .............................. & 4.500 & & & & & & & & & & \\
\hline 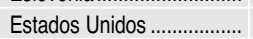 & 8.528 .252 & 4.320 & 7.290 & & & 16.020 & & 11.968 .258 & & 3.403 .080 & 4.395 .636 \\
\hline Estonia................................ & 12.000 & & & & & & & & & & \\
\hline Filipinas .............................. & & & & & & & & & & 36.407 .813 & \\
\hline 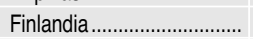 & 5.900 & & & & & & & & & 613.988 & \\
\hline Francia......................................... & 144.766 & & 184.140 & 2.863 .212 & & & & 6.976 .887 & 84.330 & 143.236 .096 & 4.087.177 \\
\hline Grecia .................................. & & & & & & & & 18.542 .253 & & & \\
\hline 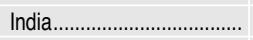 & & & & & & 6.527 & & & 1.791 .540 & 14.704 & 2.712 .717 \\
\hline Indonesia & & 784.862 & 726.660 & 960.420 & & & & & & 7.930 .731 & 85.280 \\
\hline Irak............................................ & & & 37.321 .542 & 139.007 & & & & & & & \\
\hline 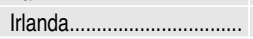 & & & & & & & & & & 44.281 & \\
\hline Israel.................................. & & & & 219.950 & & & & & & & \\
\hline Italia & 81.334 & 443.984 & 203.000 & 0 & & 680.822 & & 5.038 .247 & 1.996 .445 & 27.104 .381 & 246.798 \\
\hline 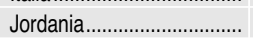 & & & & & & & & & & 808.317 & \\
\hline Kazajistán ............................ & 5.148 & & & & & & & & & 883.342 & \\
\hline Kenia & & & & & & & & & 1.352 .795 & & \\
\hline Kuwait............................... & & & 259.000 & & & & & & & & \\
\hline Luxemburgo......................... & & & & & & & & & & 125.255 & \\
\hline 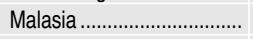 & & & & & & & & & & 165.322 .243 & \\
\hline Marruecos............................. & & & 13.324 .858 & 290.700 & & & & 834.580 & & 789.751 & 465.310 \\
\hline México ……............................... & 15.468 & & & & & & & & & 37.057 .483 & \\
\hline Noruega................................ & 6.650 & & & 499.000 & & 23.063 & & 306.000 & & & \\
\hline Nueva Zelanda ...................... & 40.700 & & 1.578 .400 & 4.641 .250 & & & & & & & \\
\hline Omán.................................. & & & & & & & & & & 79.279 .691 & \\
\hline Países Bajos ....................... & & & & & & & & & 255.814 & & \\
\hline Pakistán............................... & & & & & & 52.409 & & & & 204.478 & 181.982 \\
\hline 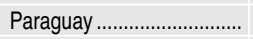 & 0 & & 1.000 & & & & & & & & \\
\hline 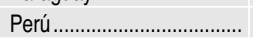 & & 5.817 .560 & & & 384.443 & 116.716 & & & & 94.120 & 1.211 .100 \\
\hline Polonia............................... & & & & & & & & & & 6.138 & \\
\hline 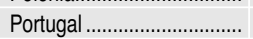 & 271.277 & 3.302 & & 236.017 & & & & & & 38.900 & \\
\hline Reino Unido........................ & 37.120 & & 189.200 & 10.712 .000 & & 0 & & 0 & & 210.849 .002 & 2.708 .767 \\
\hline Rep. Checa ......................... & 1.020 & & 300.030 & & & & & & & 3.229 .587 & \\
\hline Rumanía .............................. & 12.600 & & & & & 2.656 & & & & & \\
\hline Serbia & 12.013 & & & & & & & & & & \\
\hline 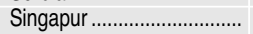 & & & & & & 2.171 .350 & & & & & \\
\hline 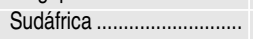 & 7.600 & & & & & & & & & 783.777 & \\
\hline Suecia................................ & 8.500 & & & & & & & & & & \\
\hline Suiza................................ & 7.600 & & 9.133 & & & & & & & 0 & \\
\hline 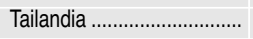 & & & 412.416 & & & & & & 853.600 & & \\
\hline
\end{tabular}




\section{EXPORTACIONES ESPAÑOLAS DE MATERIAL DE DEFENSA...}

\begin{tabular}{|c|c|c|c|c|c|c|c|c|c|c|c|}
\hline \multirow{3}{*}{ País } & 3. EX & ORTACIO & $\begin{array}{r}\text { IES REALI } \\
\text { PRI }\end{array}$ & $\begin{array}{l}\text { ADAS DE } \\
\text { IER SEME }\end{array}$ & $\begin{array}{l}\text { ATERIAL } \\
\text { RE } 2015\end{array}$ & $\begin{array}{l}\text { DE DEFE } \\
\text { (Continua }\end{array}$ & $\begin{array}{l}\text { A POR } \\
\text { ón) }\end{array}$ & PAÍS Y AR & ÍCULO & & \\
\hline & \multicolumn{11}{|c|}{ Artículo y valor en euros } \\
\hline & 1 & 2 & 3 & 4 & 5 & 6 & 7 & 8 & 9 & 10 & 11 \\
\hline 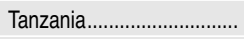 & 1.600 & & 870 & & & & & & & & \\
\hline 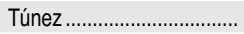 & & & & 9.156 .000 & & & 96.422 & & & & \\
\hline 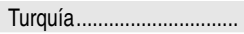 & & & & & & & & & 207.215 & 13.971 .467 & 970.000 \\
\hline Ucrania............................... & 20.290 & & & & & & & & & & \\
\hline 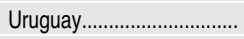 & & & 318.800 & & & & & & & & 173.479 \\
\hline Uzbekistán ......................... & & & & & & & & & & 8.150 .345 & \\
\hline 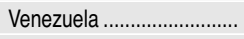 & & & & & & & & & 13.142 .752 & & \\
\hline Vietnam & & & & & 393.000 & & & & & 59.360 .935 & \\
\hline TOTAL & 9.494 .906 & 7.068 .028 & 59.909 .735 & 36.133 .746 & 997.443 & 8.877 .469 & 96.422 & 43.761 .404 & 23.458.347 & 1.497 .460 .377 & 24.007 .728 \\
\hline
\end{tabular}




\section{Subdirección General de Comercio Internacional de Material de Defensa y Doble Uso}

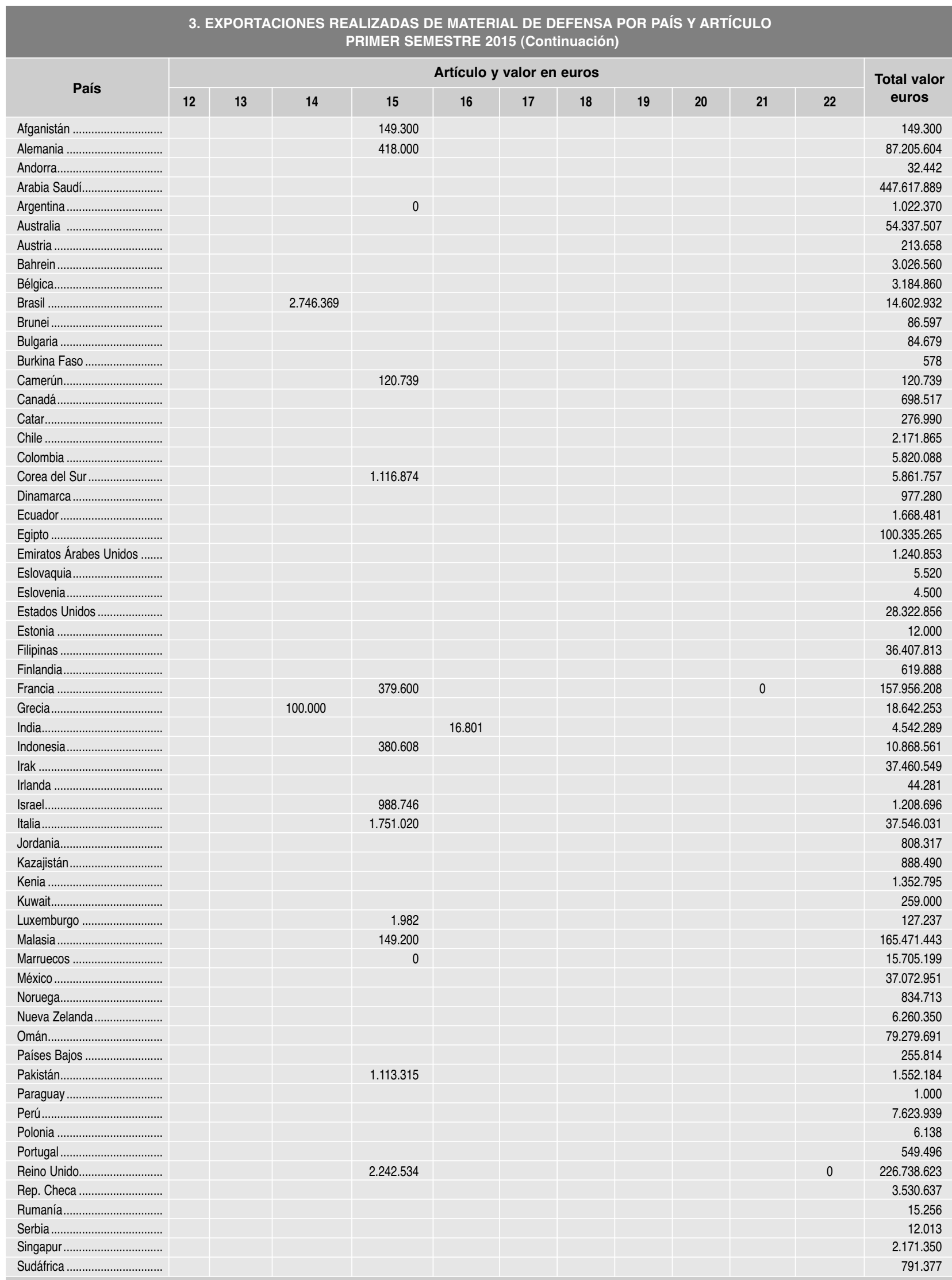

Fuente: Departamento de Aduanas e IIEE (Ministerio de Hacienda y AAPP) y empresas exportadoras. Elaboración: Subdirección General de Comercio Internacional de Material de Defensa y Doble Uso (Ministerio de Economía y Competitividad). 


\section{EXPORTACIONES ESPAÑOLAS DE MATERIAL DE DEFENSA...}

3. EXPORTACIONES REALIZADAS DE MATERIAL DE DEFENSA POR PAÍS Y ARTíCULO PRIMER SEMESTRE 2015 (Continuación)

\begin{tabular}{|c|c|c|c|c|c|c|c|c|c|c|c|c|}
\hline \multirow{2}{*}{ País } & \multicolumn{11}{|c|}{ Artículo y valor en euros } & \multirow{2}{*}{$\begin{array}{c}\text { Total valor } \\
\text { euros }\end{array}$} \\
\hline & 12 & 13 & 14 & 15 & 16 & 17 & 18 & 19 & 20 & 21 & 22 & \\
\hline 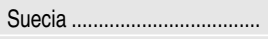 & & 1.992 .811 & & & & & & & & & & 2.001 .311 \\
\hline 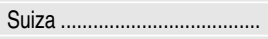 & & & & & & & & & & 2.187 .305 & & 2.204 .038 \\
\hline 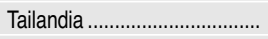 & & & & & & & & & & & & 1.266 .016 \\
\hline 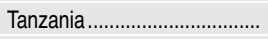 & & & & & & & & & & & & 2.470 \\
\hline 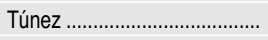 & & & & & & & & & & & & 9.252 .422 \\
\hline 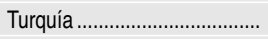 & & & & & & & & & & & & 15.148 .682 \\
\hline 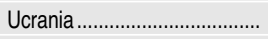 & & & & & & & & & & & & 20.290 \\
\hline 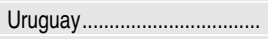 & & & & 82.114 & & & & & & & & 574.393 \\
\hline Uzbekistán............................. & & & & & & & & & & & & 8.150 .345 \\
\hline 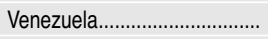 & & & & & & & & & & & & 13.142 .752 \\
\hline Vietnam & & & & & & & & & & & & 59.753 .935 \\
\hline TOTAL & & 1.992 .811 & 2.846 .369 & 8.894 .032 & 16.801 & & & & & 2.187.305 & 0 & 1.727 .202 .923 \\
\hline
\end{tabular}




\section{Subdirección General de Comercio Internacional de Material de Defensa y Doble Uso}

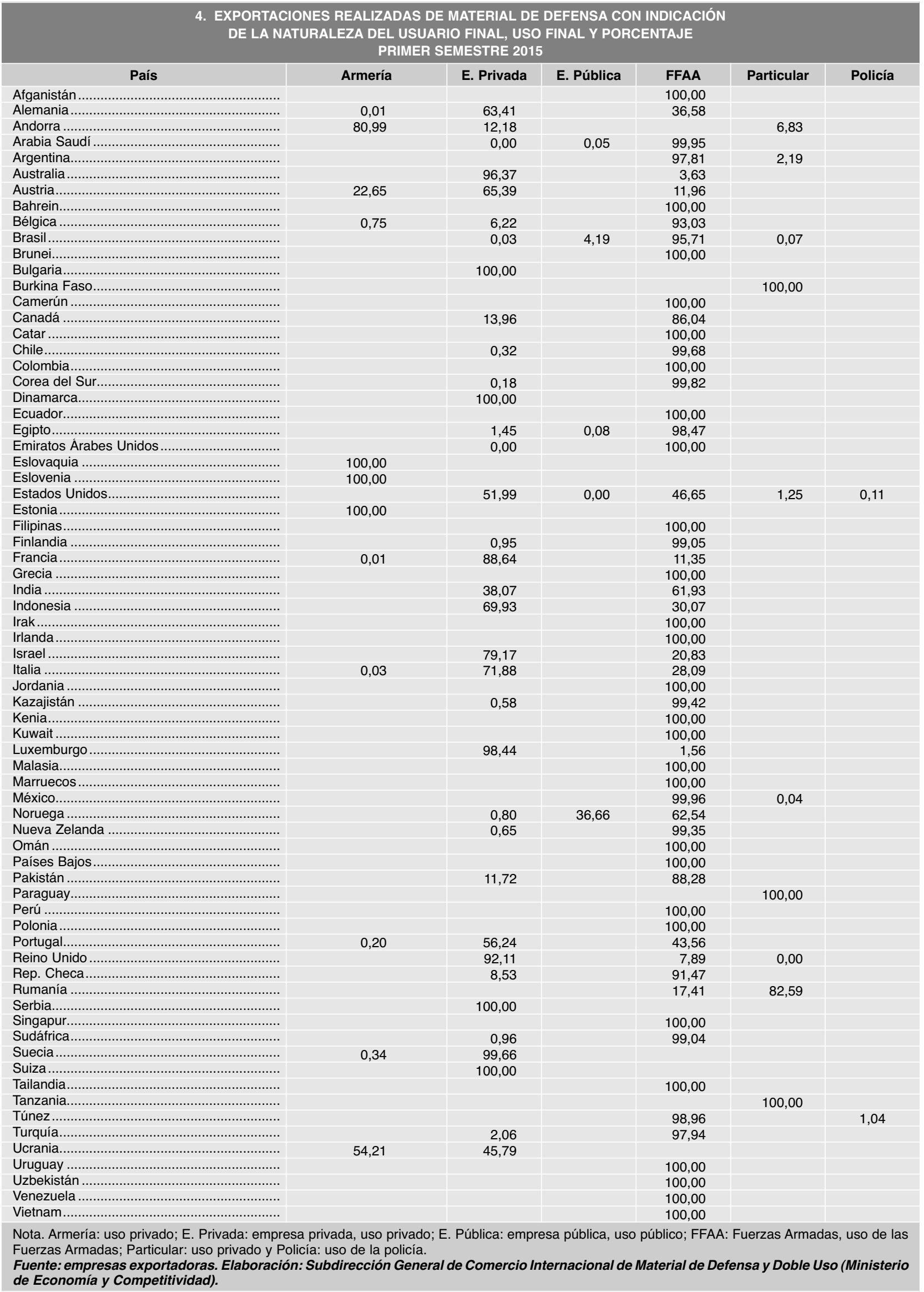




\begin{tabular}{|c|c|c|c|c|c|c|c|c|c|}
\hline \multicolumn{10}{|c|}{$\begin{array}{l}\text { 5. EXPORTACIONES REALIZADAS DE MATERIAL DE DEFENSA SUPERIORES A } 10 \text { MILLONES DE EUROS } \\
\text { PRIMER SEMESTRE } 2015\end{array}$} \\
\hline \multicolumn{2}{|l|}{ País } & \multicolumn{6}{|c|}{ Material } & \multicolumn{2}{|c|}{ Valor euros } \\
\hline \multirow{2}{*}{\multicolumn{2}{|c|}{ 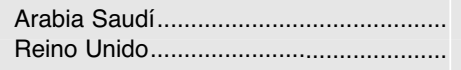 }} & \multirow{3}{*}{\multicolumn{6}{|c|}{$\begin{array}{l}2 \text { aviones para reabastecimiento en vuelo, repuestos y asistencia técnica } \\
1 \text { avión de transporte militar, repuestos y asistencia técnica } \\
1 \text { avión de transporte militar, repuestos y asistencia técnica }\end{array}$}} & \multicolumn{2}{|c|}{447.393 .033} \\
\hline & & & & & & & & \multicolumn{2}{|c|}{178.300 .116} \\
\hline \multicolumn{2}{|c|}{ 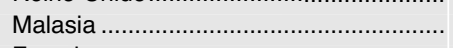 } & & & & & & & \multicolumn{2}{|c|}{165.322 .243} \\
\hline \multirow{2}{*}{\multicolumn{2}{|c|}{ Francia }} & \multicolumn{6}{|c|}{1 avión de transporte militar, repuestos y asistencia técnica } & \multicolumn{2}{|c|}{ 133.329.362 } \\
\hline & & \multicolumn{6}{|c|}{4 aviones de transporte militar, repuestos y asistencia técnica } & \multicolumn{2}{|c|}{96.662 .716} \\
\hline Omán & $\ldots \ldots \ldots$ & \multicolumn{6}{|c|}{1 avión de transporte militar, repuestos y asistencia técnica } & \multicolumn{2}{|c|}{79.279 .691} \\
\hline Vietnam ......................................... & $\ldots \ldots \ldots$ & \multicolumn{6}{|c|}{2 aviones de transporte militar, repuestos y asistencia técnica } & \multicolumn{2}{|c|}{59.329 .960} \\
\hline Australia...................................... & ........... & \multicolumn{6}{|c|}{ Partes y piezas para el montaje de aeronaves } & \multicolumn{2}{|c|}{51.280 .169} \\
\hline Filipinas ...................................... & & \multicolumn{6}{|c|}{1 avión de transporte militar, repuestos y asistencia técnica } & \multicolumn{2}{|c|}{36.407 .813} \\
\hline Alemania...... & & \multicolumn{6}{|c|}{ Partes y piezas para el montaje de aeronaves } & \multicolumn{2}{|c|}{33.738 .961} \\
\hline México ......... & & \multicolumn{6}{|c|}{1 avión de transporte militar, repuestos y asistencia técnica } & \multicolumn{2}{|c|}{33.319 .496} \\
\hline Irak............. & & \multicolumn{6}{|c|}{ Munición de artillería } & \multicolumn{2}{|c|}{23.945 .941} \\
\hline 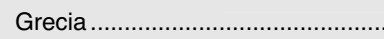 & & \multicolumn{6}{|c|}{ Combustible militar } & 18.5 & 2.253 \\
\hline Turquía ....................................... & $\ldots \ldots \ldots$ & Partes & s y piezas para & el montaje de & geronaves & & & 13.6 & 0.079 \\
\hline Estados Unidos ............................. & ............. & Combi & ustible militar & & & & & 11.6 & 1.661 \\
\hline Venezuela ....................................... & ............ & Sistem & na de artillería & ametrallado & ligera para u & o naval & & $10.1 \varepsilon$ & 9.719 \\
\hline TOTAL & . & ............ & . & ................. & …......... & . & .......... & 1.392.3 & 3.213 \\
\hline $\begin{array}{l}\text { Fuente: Departamento de Aduan } \\
\text { de Material de Defensa y Doble }\end{array}$ & nas e II & $\begin{array}{l}\text { IEE (Mi } \\
\text { Minister }\end{array}$ & $\begin{array}{l}\text { inisterio de } \mathrm{H} \\
\text { rio de Econor }\end{array}$ & $\begin{array}{l}\text { ienda y } A A I \\
\text { y Competi }\end{array}$ & $\begin{array}{l}\text { y empre } \\
\text { dad). }\end{array}$ & exportad & Elabor & Subdirecc & n General \\
\hline 6. EXPORTACIC & ONES F & REALIZ & $\begin{array}{r}\text { ZADAS EN LO: } \\
\text { PI }\end{array}$ & $\begin{array}{l}\text { PROGRAM } \\
\text { IMER SEME }\end{array}$ & $\begin{array}{l}\text { MÁS REL } \\
\text { RE } 2015\end{array}$ & NTES DE & TERIA & EENSA & \\
\hline País & A40 & 0OM & EF-2000 & IRIS-T & METEOR & MIDS & MRTT & TIGRE & $\begin{array}{l}\text { Total valor } \\
\text { euros }\end{array}$ \\
\hline Alemania ................................... & 42.23 & 31.082 & 34.247 .244 & 1.337 .013 & 544.243 & 69.000 & & 3.018 .447 & 81.447 .029 \\
\hline 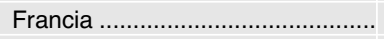 & 139.57 & 76.455 & & & & 299.000 & & 2.177 .453 & 142.052 .908 \\
\hline 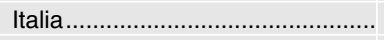 & & & 19.836 .879 & & & 1.449 .492 & & & 21.286 .371 \\
\hline Reino Unido .................................. & 146.63 & 37.306 & 58.231 .756 & & 8.787 .929 & & 3.334 .1 & & 216.991.137 \\
\hline Turquía ....................................... & 13.97 & 71.467 & & & & & & & 13.971 .467 \\
\hline TOTAL. & 342.41 & 16.310 & 112.315 .879 & 1.337 .013 & 9.332 .172 & 1.817 .492 & 3.334 .1 & 5.195 .900 & 475.748 .912 \\
\hline
\end{tabular}

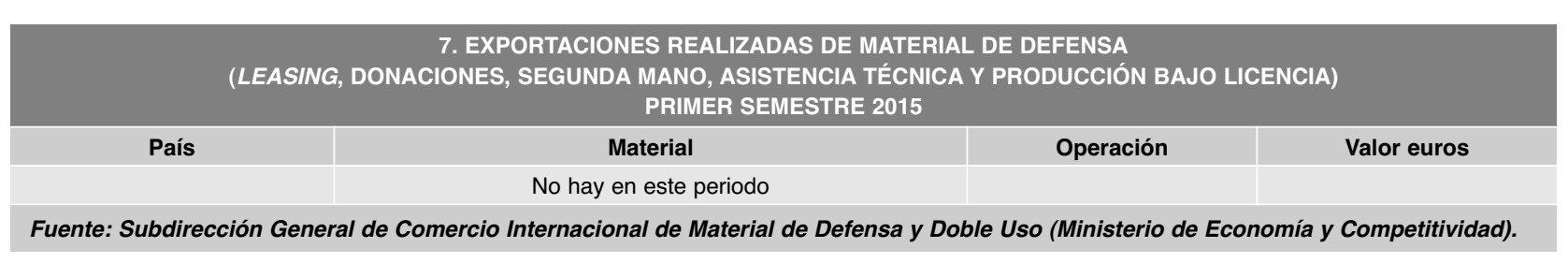

\begin{tabular}{|c|c|c|c|c|}
\hline & \multicolumn{2}{|c|}{ 8. DENEGACIONES APLICADAS EN LA EXPORTACIÓN DE MATERIAL DE DEFENSA } \\
\hline PRIMER SEMESTRE 2015
\end{tabular}




\section{Subdirección General de Comercio Internacional de Material de Defensa y Doble Uso}

\section{DESCRIPCIÓN DE LOS 22 ARTículOS DE LA RELACIÓN DE MATERIAL DE DEFENSA (REAL DECRETO 679/2014, DE 1 DE AGOSTO}

\begin{tabular}{|c|c|}
\hline Categoría & Descripción \\
\hline 1 & $\begin{array}{l}\text { Armas con cañón de ánima lisa con un calibre inferior a } \\
20 \mathrm{~mm}\end{array}$ \\
\hline 2 & $\begin{array}{l}\text { Armas con cañón de ánima lisa con un calibre igual o su- } \\
\text { perior a } 20 \mathrm{~mm}\end{array}$ \\
\hline
\end{tabular}

perior a $20 \mathrm{~mm}$

\section{Relación de materiales incluidos}

Fusiles, revólveres, pistolas, fusiles ametralladoras, silenciadores, cargadores para estas armas, visores ópticos y apagafogonazos

Armas de fuego (incluidas las piezas de artillería), rifles, obuses, cañones, morteros, armas contracarro, lanzaproyectiles, lanzallamas, cañones sin retroceso, dispositivos para la reducción de la firma, proyectores o generadores militares para humos, gases, material pirotécnico, visores y cargadores para estas armas

Municiones para las armas sometidas a control por los artículos 1, 2 o 12 Dispositivos para el armado de los cebos, se incluyen las vainas, los eslabones, las cintas, las fuentes de alimentación de elevada potencia de salida y las submuniciones

Bombas, torpedos, granadas, botes de humo, cohetes, minas, misiles, cargas de profundidad, cargas de demolición, «productos pirotécnicos», cartuchos y simuladores, granadas fumígenas, bombas incendiarias, toberas de cohetes de misiles y puntas de ojiva de vehículos de reentradas

Visores de armas, ordenadores de bombardeo, equipo de puntería para cañones, sistemas de control para armas y sistemas de adquisición de datos, de vigilancia o rastreo, reconocimiento o identificación

Carros y otros vehículos militares armados y vehículos militares equipados con soportes para armas o equipos para el sembrado de minas, vehículos blindados, vehículos anfibios, los neumáticos a prueba de bala

Agentes químicos, biológicos y materiales radiactivos, agentes nerviosos, vesicantes, gases lacrimógenos, agentes antidisturbios

Explosivos, propulsantes, productos pirotécnicos, combustibles y sustancias relacionadas, percloratos, cloratos y cromatos, oxidantes, aglomerantes, aditivos y precursores

Buques de superficie o subacuáticos, equipos navales, motores y sistemas de propulsión, redes antisubmarinos y antitorpedos

Aeronaves, vehículos más ligeros que el aire tripulados, aeronaves no tripuladas, motores aeronáuticos, vehículos aéreos teledirigidos, abastecedores de combustible, equipos de respiración presurizados, paracaídas y parapentes

Equipos de contramedidas y contra-contramedidas electrónicas, material acústico submarino, equipos de seguridad de los datos, equipos que utilicen cifrado, equipos de guiado, navegación y transmisiones

Sistemas de armas de energía cinética, instalaciones de ensayo y de evaluación y modelos de prueba, sistemas de propulsión, sistemas de búsqueda de objetivos, de guiado o de propulsión derivada para proyectiles

Planchas de blindaje, construcciones de materiales metálicos o no, cascos militares, trajes blindados o prendas de protección

Entrenadores de ataque, de vuelo, de blancos radar, de guerra antisubmarina, para el lanzamiento de misiles, de generación de imagen

Registradores y equipos de proceso de imagen, cámaras, equipo fotográfico, equipo para la intensificación de imágenes, equipo de formación de imagen de infrarrojos o térmica, equipo sensor de imagen por radar

Productos semielaborados que sean identificables por la composición del material, geometría o función

Aparatos autónomos de inmersión y natación subacuática, aparatos de circuito cerrado y semicerrado, robots, transbordadores

Instalaciones de ensayo ambiental, nitruradores de tipo continuo, equipos o aparatos de ensayo por centrifugación, prensas extruidoras de husillo

Sistemas láser, de haces de partículas, de radiofrecuencia, aceleradores de partículas

Equipos diseñados especialmente o configurados para ser instalados en vehículos para aplicaciones militares terrestres, marítimas, aeronáuticas o espaciales, equipos eléctricos superconductores

Equipo lógico (software) para la modelización, la simulación o la evaluación de sistemas de armas militares o de simulación de escenarios de operaciones militares, para las aplicaciones de mando, comunicaciones, control e inteligencia

Tecnología para el desarrollo, producción o utilización de los materiales sometidos a control

Fuente: Subdirección General de Comercio Internacional de Material de Defensa y Doble Uso (Ministerio de Economía y Competitividad). 
EXPORTACIONES ESPAÑOLAS DE MATERIAL DE DEFENSA...

10. EXPORTACIONES AUTORIZADAS DE OTRO MATERIAL (ANTIDISTURBIOS) POR PAÍS Y NÚMERO DE LICENCIAS PRIMER SEMESTRE 2015

\begin{tabular}{|c|c|c|}
\hline País & $\mathrm{N}^{\circ}$ Licencias & Valor euros \\
\hline 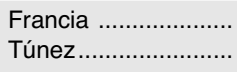 & $\begin{array}{l}2 \\
1\end{array}$ & $\begin{array}{r}71.952 \\
1202.623\end{array}$ \\
\hline TOTAL & 3 & 274.575 \\
\hline
\end{tabular}

Fuente: empresas exportadoras. Elaboración: Subdirección General de Comercio Internacional de Material de Defensa y Doble Uso (Ministerio de Economía y Competitividad).

12. EXPORTACIONES REALIZADAS DE OTRO MATERIAL (ANTIDISTURBIOS) CON INDICACIÓN DE LA NATURALEZA DEL USUARIO FINAL Y EL USO FINAL PRIMER SEMESTRE 2015

\begin{tabular}{|c|c|c|c|}
\hline Pais & Usuario final & Uso final & Porcentaje \\
\hline Perú .................... & FFAA & Público & 100 \\
\hline Portugal........... & Policía & Público & 100 \\
\hline Togo ................. & Policía & Público & 100 \\
\hline Túnez ............... & Policía & Público & 100 \\
\hline
\end{tabular}

Fuente: Empresas exportadoras. Elaboración: Subdirección General de Comercio Internacional de Material de Defensa y Doble Uso (Ministerio de Economía y Competitividad).

\begin{tabular}{|c|c|c|}
\hline \multicolumn{3}{|c|}{$\begin{array}{l}\text { 11. EXPORTACIONES REALIZADAS DE OTRO MATERIAL } \\
\text { (ANTIDISTURBIOS) POR PAIS } \\
\text { PRIMER SEMESTRE } 2015\end{array}$} \\
\hline País & Material & $\begin{array}{l}\text { Valor } \\
\text { euros }\end{array}$ \\
\hline Perú .............. & $\begin{array}{l}\text { Artificios lacrimógenos y fumígenos, des- } \\
\text { pieces y materia prima para la fabricación } \\
\text { de artificios pirotécnicos no letales y car- } \\
\text { tuchos lacrimógenos }\end{array}$ & 950.849 \\
\hline Portugal ...... & $\begin{array}{l}\text { Bocachas, artificios triples lacrimógenos, } \\
\text { cartuchos propulsores y botes de humo }\end{array}$ & 13.015 \\
\hline Togo ............ & $\begin{array}{l}\text { Botes lacrimógenos, cartuchos, artificios } \\
\text { de luz y sonido y bocachas }\end{array}$ & 0 \\
\hline Túnez.......... & $\begin{array}{l}\text { Granadas de mano de gas lacrimógeno y } \\
\text { aerosoles de defensa personal }\end{array}$ & 207.200 \\
\hline \multicolumn{2}{|c|}{ TOTAL. } & 1.171 .064 \\
\hline $\begin{array}{l}\text { Fuente: De } \\
\text { y AAPP) } \\
\text { General d } \\
\text { Doble Uso }\end{array}$ & $\begin{array}{l}\text { amento de Aduanas e IIEE (Ministerio } \\
\text { ipresas exportadoras. Elaboración: } \\
\text { omercio Internacional de Material a } \\
\text { nisterio de Economía y Competitivida }\end{array}$ & $\begin{array}{l}\text { Hacienda } \\
\text { bdirección } \\
\text { Defensa y }\end{array}$ \\
\hline
\end{tabular}

\begin{tabular}{|c|c|c|c|}
\hline \multicolumn{4}{|c|}{$\begin{array}{l}\text { 13. EXPORTACIONES REALIZADAS DE OTRO MATERIAL } \\
\text { (ANTIDISTURBIOS) (LEASING, DONACIONES, } \\
\text { SEGUNDA MANO, ASISTENCIA TÉCNICA Y } \\
\text { PRODUCCIÓN BAJO LICENCIA) } \\
\text { PRIMER SEMESTRE } 2015\end{array}$} \\
\hline País & Producto & Operación & $\begin{array}{l}\text { Valor } \\
\text { euros }\end{array}$ \\
\hline Togo & Botes lacrimógenos, cartuchos, artificios de luz y sonido y bocachas & Donación & 0 \\
\hline
\end{tabular}

14. DENEGACIONES APLICADAS EN LA EXPORTACIÓN DE OTRO MATERIAL (ANTIDISTURBIOS) PRIMER SEMESTRE 2015

\begin{tabular}{|c|c|c|c|}
\hline \multicolumn{4}{|c|}{$\begin{array}{l}\text { 14. DENEGACIONES APLICADAS EN LA EXPORTACIÓN DE OTRO MATERIAL (ANTIDISTURBIOS) } \\
\text { PRIMER SEMESTRE } 2015\end{array}$} \\
\hline País & Número & Producto & Motivo \\
\hline \multicolumn{4}{|c|}{ No ha habido en este periodo } \\
\hline
\end{tabular}

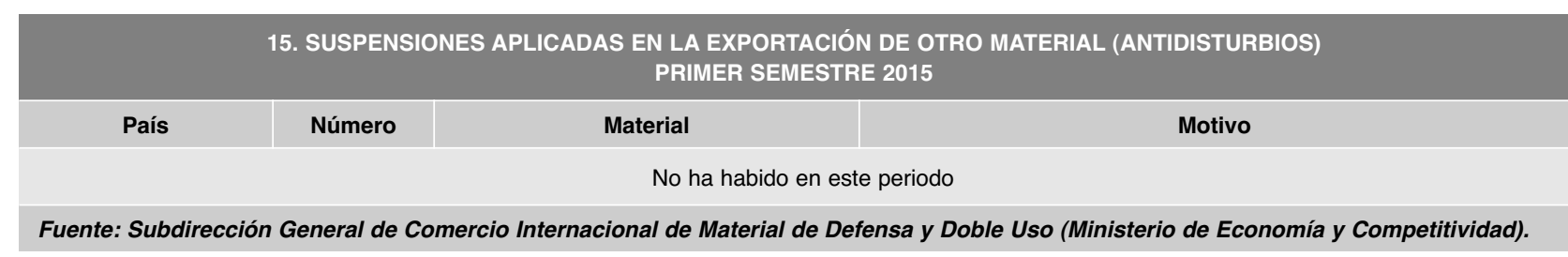




\section{Subdirección General de Comercio Internacional de Material de Defensa y Doble Uso}

16. EXPORTACIONES AUTORIZADAS DE OTRO MATERIAL (ARMA DE CAZA Y TIRO DEPORTIVO) POR PAÍS Y NÚMERO DE LICENCIAS PRIMER SEMESTRE 2015

\begin{tabular}{|c|c|c|c|c|c|}
\hline País & $\mathrm{N}^{\circ}$ Licencias & Valor euros & País & $\mathrm{N}^{\circ}$ Licencias & Valor euros \\
\hline Andorra & 3 & 225 & 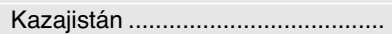 & 2 & 4.550 .000 \\
\hline 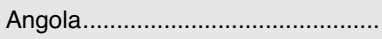 & 1 & 1.200 .000 & 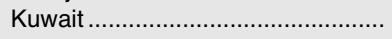 & 1 & 140.000 \\
\hline Argentina $\ldots \ldots \ldots \ldots \ldots \ldots \ldots \ldots \ldots \ldots \ldots \ldots \ldots \ldots \ldots \ldots \ldots \ldots$ & 14 & 470.219 & 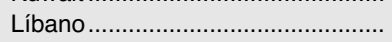 & 8 & 272.573 \\
\hline 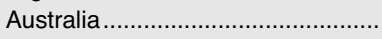 & 4 & 4.650 .000 & 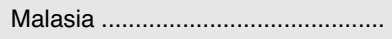 & 1 & 100.000 \\
\hline Bangladesh ................... & 2 & 111.500 & 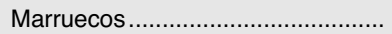 & 3 & 490.000 \\
\hline 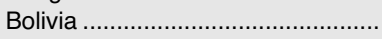 & 2 & 400.000 & 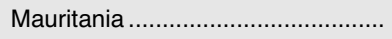 & 5 & 1.222 .400 \\
\hline Brasil & 1 & 100 & 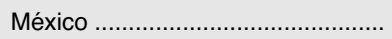 & 1 & 520.000 \\
\hline 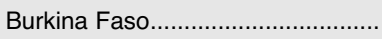 & 1 & 83.173 & 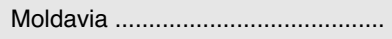 & 2 & 90.000 \\
\hline Camerún & 4 & 5.215 .000 & 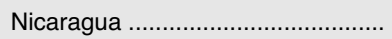 & 5 & 553.000 \\
\hline 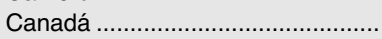 & 1 & 44.810 & Noruega ................................... & 3 & 1.701 .600 \\
\hline 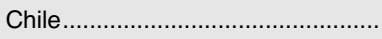 & 9 & 754.970 & Pakistán ........................................... & 2 & 80.000 \\
\hline 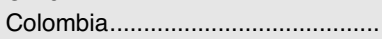 & 2 & 321.000 & 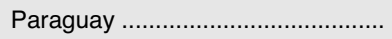 & 8 & 801.000 \\
\hline 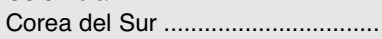 & 5 & 192.070 & 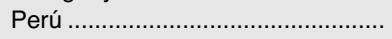 & 2 & 347.000 \\
\hline 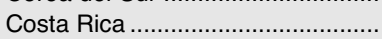 & 1 & 90.000 & 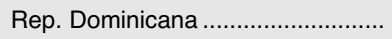 & 2 & 530.000 \\
\hline 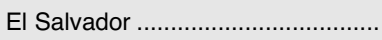 & 3 & 98.000 & Rusia & 4 & 1.296 .105 \\
\hline 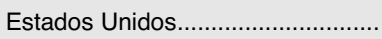 & 25 & 22.680 .979 & 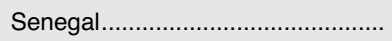 & 1 & 120.000 \\
\hline 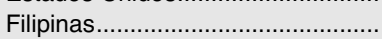 & 1 & 500.000 & Sudáfrica..................................... & 1 & 700.000 \\
\hline 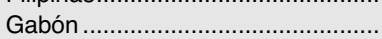 & 1 & 190.000 & 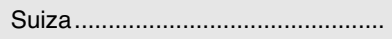 & 1 & 54.200 \\
\hline Ghana & 1 & 600.000 & 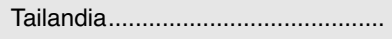 & 4 & 230.000 \\
\hline Guatemala & 1 & 95.000 & 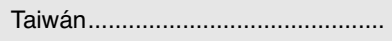 & 2 & 180.000 \\
\hline 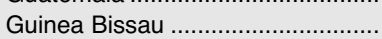 & 9 & 1.595 .699 & 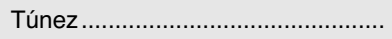 & 7 & 967.244 \\
\hline 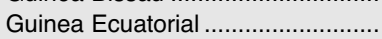 & 3 & 3.095 & 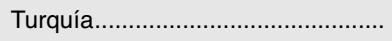 & 3 & 26.117 .500 \\
\hline 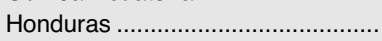 & 1 & 105.000 & 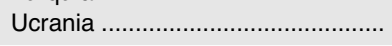 & 3 & 498.700 \\
\hline Jamaica & 1 & 115.000 & & & \\
\hline 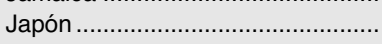 & 7 & 5.898 .380 & TOTAL & 174 & 86.975 .542 \\
\hline
\end{tabular}

Fuente: empresas exportadoras. Elaboración: Subdirección General de Comercio Internacional de Material de Defensa y Doble Uso (Ministerio de Economía y Competitividad). 
EXPORTACIONES ESPAÑOLAS DE MATERIAL DE DEFENSA...

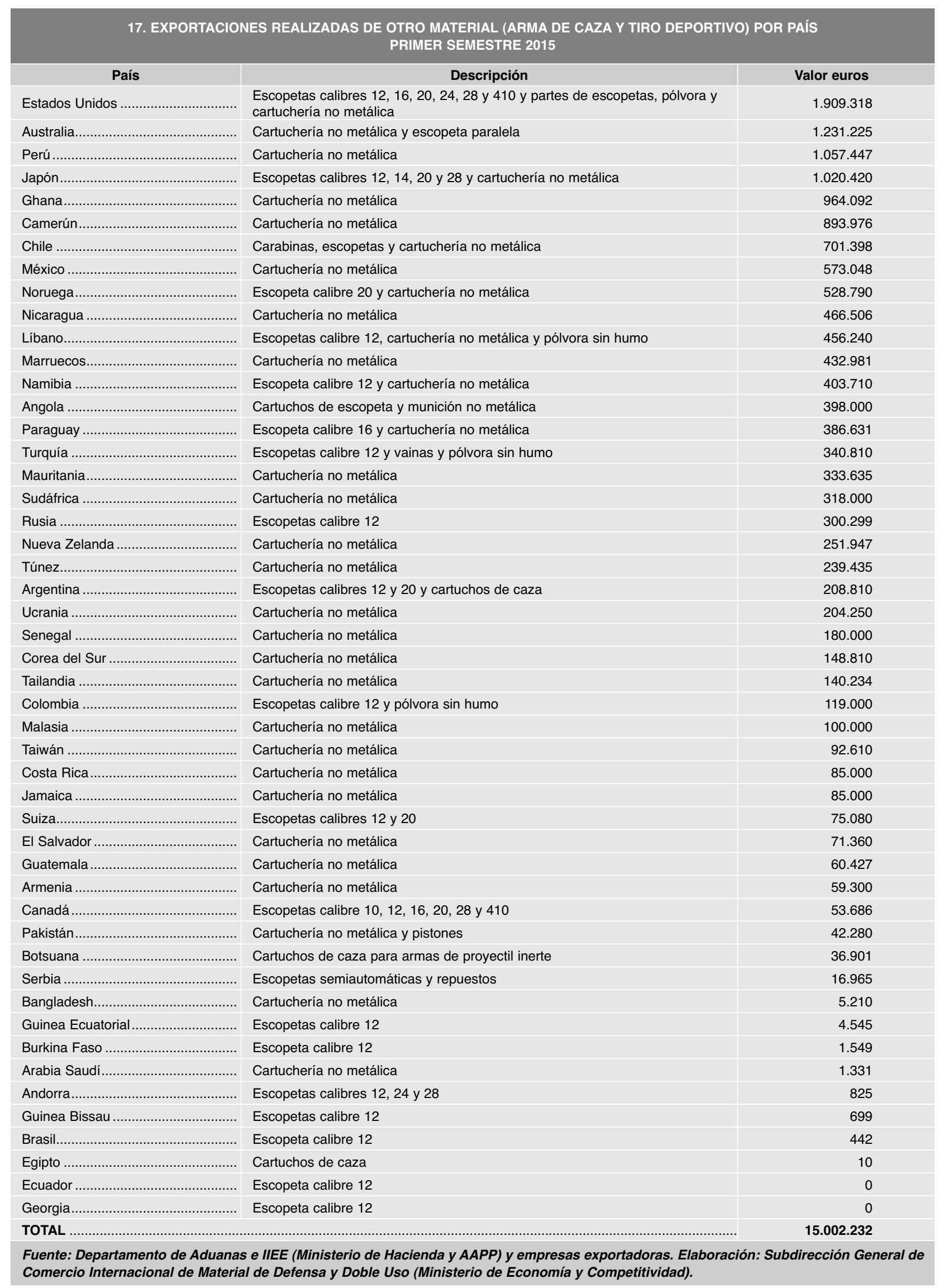




\section{Subdirección General de Comercio Internacional de Material de Defensa y Doble Uso}

\begin{tabular}{|c|c|c|c|}
\hline \multicolumn{4}{|c|}{$\begin{array}{l}\text { 18. EXPORTACIONES REALIZADAS DE OTRO MATERIAL (ARMA DE CAZA Y TIRO DEPORTIVO) } \\
\text { (LEASING, DONACIONES, SEGUNDA MANO, ASISTENCIA TÉCNICA Y PRODUCCIÓN BAJO LICENCIA) } \\
\text { PRIMER SEMESTRE } 2015\end{array}$} \\
\hline País & Producto & Operación & Valor euros \\
\hline Guinea Ecuatorial ......... & 1 escopeta calibre 12 & Donación & 0 \\
\hline 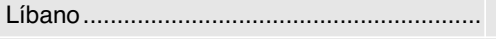 & 106 escopetas calibre 12 & Segunda mano & 20.365 \\
\hline … & .......... & ............ & 20.365 \\
\hline
\end{tabular}

19. DENEGACIONES APLICADAS EN LA EXPORTACIÓN DE OTRO MATERIAL (ARMA DE CAZA Y TIRO DEPORTIVO) PRIMER SEMESTRE 2015

\begin{tabular}{l|l|l|l} 
País & Número & Producto & Motivo
\end{tabular}

No ha habido en este periodo

Fuente: Subdirección General de Comercio Internacional de Material de Defensa y Doble Uso (Ministerio de Economía y Competitividad).

20. SUSPENSIONES APLICADAS EN LA EXPORTACIÓN DE OTRO MATERIAL (ARMA DE CAZA Y TIRO DEPORTIVO) PRIMER SEMESTRE 2015

\begin{tabular}{l|l|l|l|}
\hline País & Número & Material & Motivo
\end{tabular}

No ha habido en este periodo

Fuente: Subdirección General de Comercio Internacional de Material de Defensa y Doble Uso (Ministerio de Economía y Competitividad). 
EXPORTACIONES ESPAÑOLAS DE MATERIAL DE DEFENSA... 21. EXPORTACIONES REALIZADAS DE OTRO MATERIAL (ARMA DE CAZA Y TIRO DEPORTIVO) CON INDICACIÓN DE LA NATURALEZA
DEL USUARIO FINAL, USO FINAL Y PORCENTAJE PRIMER SEMESTRE 2015

\begin{tabular}{|c|c|c|c|c|}
\hline País & Armería & E. Privada & FFAA & Particular \\
\hline Andorra & & 0,00 & & 100,00 \\
\hline Angola & & 100,00 & & \\
\hline Arabia Saudí & & 100,00 & & \\
\hline Argentina & & 52,04 & & 47,96 \\
\hline Armenia & & 100,00 & & \\
\hline Australia & & 98,39 & & 1,61 \\
\hline Bangladesh & & 100,00 & & \\
\hline Botsuana & & 100,00 & & \\
\hline 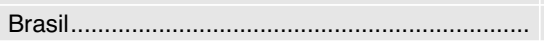 & & & & 100,00 \\
\hline Burkina Faso . & & & & 100,00 \\
\hline 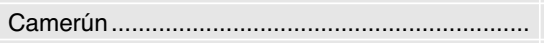 & 10,96 & 89,04 & & \\
\hline 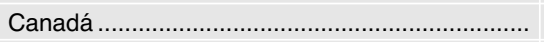 & 16,53 & 83,47 & & \\
\hline Chile & 0,07 & 99,51 & & 0,43 \\
\hline 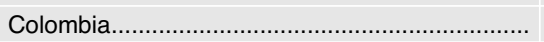 & & & 100,00 & 0,00 \\
\hline 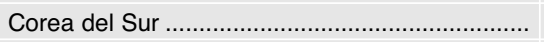 & 2,99 & 97,01 & & \\
\hline Costa Rica & & 100,00 & & \\
\hline Ecuador & & & & 100,00 \\
\hline 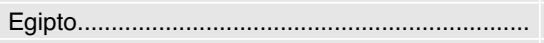 & & 100,00 & & \\
\hline 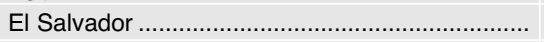 & & 100,00 & & \\
\hline 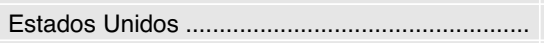 & 4,83 & 82,29 & & 12,88 \\
\hline 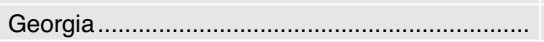 & & & & 100,00 \\
\hline 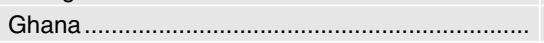 & & 100,00 & & \\
\hline Guatemala & & 100,00 & & \\
\hline 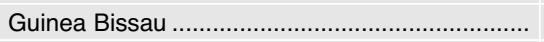 & & & & 100,00 \\
\hline 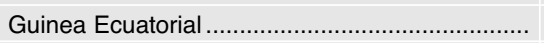 & & & & 100,00 \\
\hline 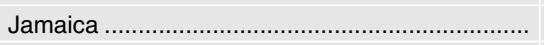 & & 100,00 & & \\
\hline Japón & & 100,00 & & \\
\hline 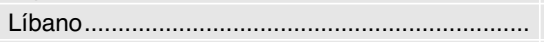 & 10,26 & 89,74 & & \\
\hline 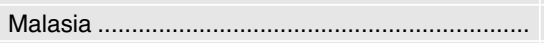 & & 100,00 & & \\
\hline Marruecos & 42,16 & 57,84 & & \\
\hline 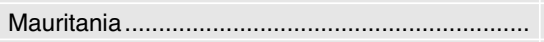 & & 100,00 & & \\
\hline México & & & 100,00 & \\
\hline Namibia. & & 100,00 & & 0,00 \\
\hline 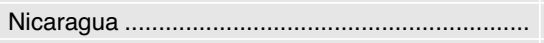 & 58,20 & 41,80 & & \\
\hline Noruega & & 100,00 & & \\
\hline 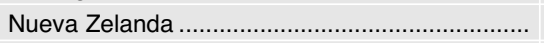 & & 100,00 & & \\
\hline Pakistán & & 100,00 & & \\
\hline 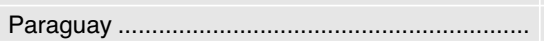 & & 100,00 & & 0,00 \\
\hline 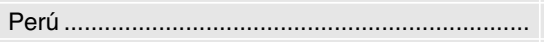 & & 100,00 & & \\
\hline 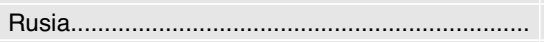 & & 100,00 & & \\
\hline 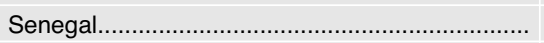 & 50,00 & 50,00 & & \\
\hline Serbia & & 100,00 & & \\
\hline 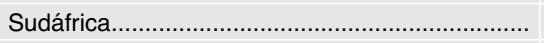 & & 100,00 & & \\
\hline 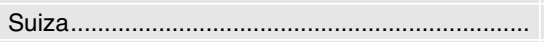 & & 72,03 & & 27,97 \\
\hline 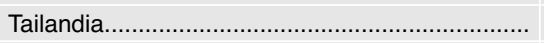 & & 100,00 & & \\
\hline 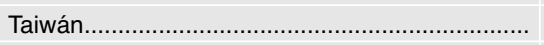 & & 100,00 & & \\
\hline 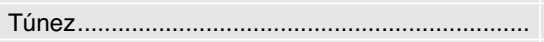 & & 100,00 & & \\
\hline Turquía & 41,32 & 58,68 & & \\
\hline 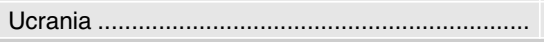 & & 100,00 & & \\
\hline
\end{tabular}




\section{Subdirección General de Comercio Internacional de Material de Defensa y Doble Uso}

22. RELACIÓN DE OTRO MATERIAL

(REAL DECRETO 679/2014, DE 1 DE AGOSTO)

ANEXO II.1. Armas de fuego, sus piezas y componentes esenciales y municiones para uso civil

1. Aquellas armas de fuego, así como sus partes y componentes esenciales y municiones, definidas en el anexo I del Reglamento (UE) N ${ }^{\circ} 258 / 2012$ del Parlamento Europeo y del Consejo, de 14 de marzo de 2012, por el que se aplica el artículo 10 del Protocolo de las Naciones Unidas contra la fabricación y el tráfico ilícitos de armas de fuego, sus piezas y componentes y municiones, que complementa la Convención de las Naciones Unidas contra la delincuencia transnacional organizada, y por el que se establecen autorizaciones de exportación y medidas de importación y tránsito para las armas de fuego, sus piezas y componentes y municiones

2. Visores y miras, telescópicos o de intensificación de luz o imagen, para armas de fuego, distintas de las incluidas en el anexo I.1 de este Real Decreto

\section{ANEXO II.2. Relación de otro material}

1. Artificios generadores, proyectores, emisores o dispensadores de humos, gases, «agentes antidisturbios" o sustancias incapacitantes

2. Lanzadores de los elementos descritos en el apartado 1 anterior

3. Equipos de luz y sonido provocadores de aturdimiento, para el control de disturbios

4. Vehículos para el control de disturbios con alguna de las siguientes características:

1. Sistemas para producir descargas eléctricas

2. Sistemas para dispensar sustancias incapacitantes

3. Sistemas para dispensar agentes antidisturbios

4. Cañones de agua

5. Esposas normales

Fuente: Subdirección General de Comercio Internacional de Material de Defensa y Doble Uso (Ministerio de Economía y Competitividad).

\begin{tabular}{|c|c|c|c|c|c|}
\hline 23. EXPORTAC & S AUTO & $\begin{array}{l}\text { DAS DE PRC } \\
\text { Y NÚMEF } \\
\text { PRIMER }\end{array}$ & $\begin{array}{l}\text { OS Y TECNOLOGÍAS DE DOBLE } \\
\text { LICENCIAS } \\
\text { STRE } 2015\end{array}$ & OF & \\
\hline País & $\mathrm{N}^{\circ}$ Licencias & Valor euros & País & $N^{\circ}$ Licencias & Valor euros \\
\hline 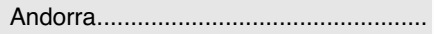 & 6 & 1.549 .171 & Malasia ........... & 1 & 0 \\
\hline 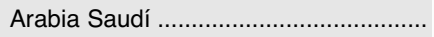 & 10 & 9.466 .167 & Malí.. & 1 & 16.740 \\
\hline Argelia & 5 & 1.153 .750 & Marruecos.... & 4 & 878.771 \\
\hline 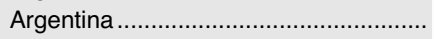 & 5 & 274.507 & México ............ & 8 & 71.192 .649 \\
\hline 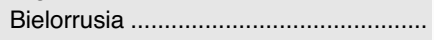 & 3 & 0 & Nicaragua & 1 & 51 \\
\hline 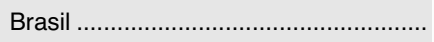 & 12 & 37.653 .996 & 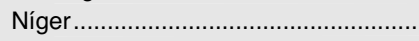 & 1 & 60.000 \\
\hline Chile .................... & 1 & 16.000 & Omán................. & 1 & 1.330 .497 \\
\hline Colombia ........................ & 14 & 5.358 .075 & Panamá ............... & 1 & 1.850 \\
\hline Corea del Sur ................. & 15 & 384.850 & Paraguay ............. & 3 & 124.704 \\
\hline Costa Rica........................ & 1 & 100.000 & Perú ....................... & 4 & 356.790 \\
\hline 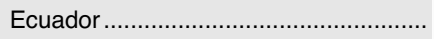 & 1 & 345 & Reino Unido... & 1 & 155.000 \\
\hline 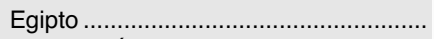 & 2 & 552 & R.P. China........... & 22 & 15.533 .118 \\
\hline Emiratos Árabes Unidos ......................... & 4 & 672.708 & Rusia ............... & 3 & 2.895 .000 \\
\hline Filipinas ............................................ & 2 & 205.419 & Senegal & 2 & 740 \\
\hline 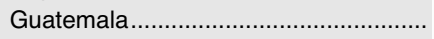 & 1 & 34.000 & Serbia ................. & 1 & 52.081 \\
\hline 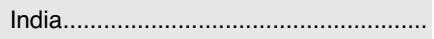 & 12 & 14.523 .173 & Sudáfrica & 3 & 3.169 .200 \\
\hline 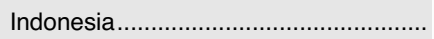 & 2 & 3.884 .892 & 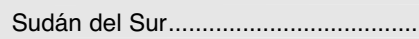 & 1 & 2.981 \\
\hline Irak ............... & 3 & 131.780 & 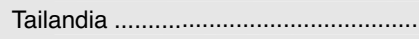 & 5 & 25.763 \\
\hline Irán .............. & 8 & 1.097.279 & Taiwán .................. & 10 & 2.909 \\
\hline Israel... & 4 & 6.282 .925 & Túnez........... & 1 & 1.840 \\
\hline Italia ................ & 1 & 0 & Turquía & 2 & 1.779 .000 \\
\hline Japón............................................. & 1 & 380.000 & 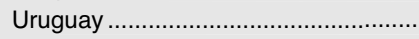 & 2 & 686.000 \\
\hline 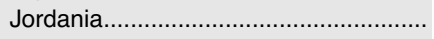 & 2 & 131.721 & Vietnam.............................................. & 4 & 41.864 \\
\hline 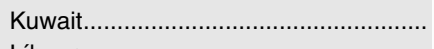 & 2 & 34.620 & TOTAL. & 201 & 181.774.671 \\
\hline 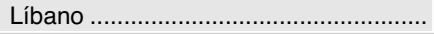 & 2 & 131.193 & & & \\
\hline
\end{tabular}

Fuente: empresas exportadoras. Elaboración: Subdirección General de Comercio Internacional de Material de Defensa y Doble Uso (Ministerio de Economía y Competitividad) 
EXPORTACIONES ESPAÑOLAS DE MATERIAL DE DEFENSA...

\begin{tabular}{|c|c|c|c|c|c|c|c|c|c|c|c|}
\hline \multirow{2}{*}{ País } & \multicolumn{10}{|c|}{ Categoría } & \multirow{2}{*}{$\begin{array}{l}\text { Valor } \\
\text { euros }\end{array}$} \\
\hline & 0 & 1 & 2 & 3 & 4 & 5 & 6 & 7 & 8 & 9 & \\
\hline Andorra ............................. & & & & & & 1.549 .171 & & & & & 1.549 .171 \\
\hline Arabia Saudí ....................... & & 946 & 9.465 .221 & & & & & & & & 9.466 .167 \\
\hline Argelia............................. & & 1.153 .750 & & & & & 0 & & & & 1.153 .170 \\
\hline Argentina ............................. & & & & 274.507 & & & & & & & 274.507 \\
\hline Bielorrusia............................. & & 0 & & & & & & & & & 0 \\
\hline 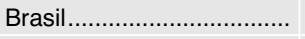 & & 20.227 .053 & 15.531 .057 & 90.338 & & 1.805 .548 & & & & & 37.653 .996 \\
\hline Chile.............................. & & & & & & 16.000 & & & & & 16.000 \\
\hline Colombia............................ & & 5.358 .075 & & & & & & & & & 5.358 .075 \\
\hline Corea del Sur ...................... & & & 297 & 378.908 & & & 5.645 & & & & 384.850 \\
\hline Costa Rica ........................ & & 100.000 & & & & & & & & & 100.000 \\
\hline Ecuador ................................ & & 345 & & & & & & & & & 345 \\
\hline Egipto.................................. & & & 552 & & & & 0 & & & & 552 \\
\hline Emiratos Arabes Unidos ... & & & 672.708 & & & & & & & & 672.708 \\
\hline 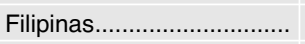 & & 919 & 204.500 & & & & & & & & 205.419 \\
\hline Guatemala ........................ & & 34.000 & & & & & & & & & 34.000 \\
\hline India ............................... & & 5.742 & 14.227 .431 & & & & & & & 290.000 & 14.523 .173 \\
\hline Indonesia .............................. & & & 3.884 .892 & & & & & & & & 3.884 .892 \\
\hline 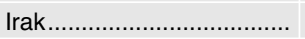 & & 131.780 & & & & & & & & & 131.780 \\
\hline Irán................................. & & & 1.097 .279 & & & & & & & & 1.097 .279 \\
\hline Israel................................ & & & 6.000 .000 & & 32.925 & & 0 & 250.000 & & & 6.282 .925 \\
\hline 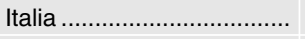 & & & & 0 & & & & & & & 0 \\
\hline Jaón ...................................... & & & & & & & & & & 380.000 & 380.000 \\
\hline 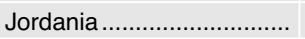 & & 131.721 & & & & & & & & & 131.721 \\
\hline Kuwait ............................. & & 420 & 34.200 & & & & & & & & 34.620 \\
\hline Líbano................................ & & 131.193 & & & & & & & & & 131.193 \\
\hline Malasia ........................... & & & & & & 0 & & & & & 0 \\
\hline Mali .................................... & & 16.740 & & & & & & & & & 16.740 \\
\hline Marruecos........................ & & 878.771 & & & & & 0 & & & & 878.771 \\
\hline México ............................. & & & 18.623 & & & 71.174 .026 & & & & 0 & 71.192 .649 \\
\hline Nicaragua ............................ & & 51 & & & & & & & & & 51 \\
\hline 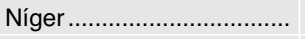 & & & & & & & & & & 60.000 & 60.000 \\
\hline 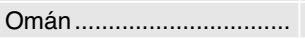 & & 1.330 .497 & & & & & & & & & 1.330 .497 \\
\hline Panamá ............................. & & 1.850 & & & & & & & & & 1.850 \\
\hline Paraguay ........................... & & 124.224 & 480 & & & & & & & & 124.704 \\
\hline Perú .................................. & 580 & 1.210 & & & & & & & & 355.000 & 356.790 \\
\hline Reino Unido .......................... & & & & & & 155.000 & & & & & 155.000 \\
\hline 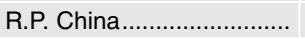 & 280.500 & & 14.987 .544 & 129.860 & & & 5.064 & & & 130.150 & 15.533 .118 \\
\hline 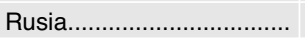 & & & 1.487 .000 & 1.346 .800 & & 61.200 & & & & & 2.895 .000 \\
\hline Senegal........................... & & 740 & & & & & & & & & 740 \\
\hline 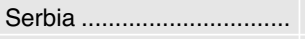 & & 52.081 & & & & & & & & & 52.081 \\
\hline Sudáfrica............................... & & 3.169 .200 & & & & & & & & & 3.169 .200 \\
\hline Sudan del Sur..................... & & & & & & 2.981 & & & & & 2.981 \\
\hline Tailandia................................ & & 5.045 & 20.718 & & & & & & & & 25.763 \\
\hline Taiwán................................ & & 2.909 & 0 & & & & & & & & 2.909 \\
\hline Túnez................................ & & 1.840 & & & & & & & & & 1.840 \\
\hline Turquía................................. & & & 1.310 .000 & & & 469.000 & & & & & 1.779 .000 \\
\hline Uruguay ............................ & & 686.000 & & & & & & & & & 686.000 \\
\hline 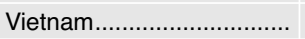 & & 793 & 41.071 & & & & & & & & 41.864 \\
\hline TOTAL & 281.080 & 33.547.895 & 68.983 .573 & 2.220 .413 & 32.925 & 75.232 .926 & 10.709 & 250.000 & & 1.215 .150 & 181.774.671 \\
\hline
\end{tabular}




\section{Subdirección General de Comercio Internacional de Material de Defensa y Doble Uso}

25. EXPORTACIONES REALIZADAS DE PRODUCTOS Y TECNOLOGÍAS DE DOBLE USO POR PAÍS Y CATEGORÍA PRIMER SEMESTRE 2015

\begin{tabular}{|c|c|c|c|c|c|c|c|c|c|c|c|}
\hline \multirow{2}{*}{ País } & \multicolumn{10}{|c|}{ Categoría } & \multirow{2}{*}{$\begin{array}{l}\text { Valor } \\
\text { euros }\end{array}$} \\
\hline & 0 & 1 & 2 & 3 & 4 & 5 & 6 & 7 & 8 & 9 & \\
\hline Andorra...... & & & & & & 252.827 & & & & & 252.827 \\
\hline Arabia Saudí........................ & & 5.255 .126 & 1.646 .883 & & & 1.526 .318 & & & & & 8.428 .327 \\
\hline Argelia................................. & & 38.113 & & & & & & & & & 38.113 \\
\hline Argentina ........................... & & 474.483 & & 898.113 & & & & & & & 1.372 .596 \\
\hline Australia ................................ & & 601 & 0 & & & 5.372 & 995 & & & & 6.968 \\
\hline Bangladesh.......................... & & & 1.000 .000 & & & & & & & & 1.000 .000 \\
\hline Brasil.................................. & & 393.132 & 4.697 .708 & 27.878 & & & & & & & 5.118 .718 \\
\hline Canadá ................................. & 1.478 .881 & & & & & & & & & 29.190 & 1.508 .071 \\
\hline Chile.................................. & & 12.330 & & & & 4.280 & & & & & 16.610 \\
\hline Colombia.............................. & & 165.688 & & & & & & & & & 165.688 \\
\hline Corea del Sur ...................... & & & 297 & 249.719 & & 20 & 5.645 & & & & 255.681 \\
\hline Ecuador ............................. & & 345 & & & & & & & & & 345 \\
\hline Emiratos Árabes Unidos... & & & 6.722 .043 & 87.736 & & & & & & & 6.809 .779 \\
\hline Estados Unidos ................. & 780.547 & 150.126 & 13.958 .990 & 563.558 & & 14.028 & 24.565 & & & 985.350 & 16.477 .164 \\
\hline Filipinas................................ & & 919 & 204.500 & & & & & & & & 205.419 \\
\hline Francia................................ & & 0 & & & & & & & & & 0 \\
\hline Hong Kong (R.P. China) ... & & & & & & & & & & 18.350 & 18.350 \\
\hline India ................................. & & 5.742 & 6.210 & 209.968 & & 231 & & & & & 222.151 \\
\hline 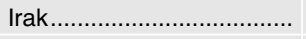 & & 534 & & & & & & & & & 534 \\
\hline 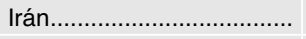 & & & 5.904 .640 & & & & & & & & 5.904 .640 \\
\hline Israel .................................. & & & & & 32.925 & 3.800 & & 130.587 & & & 167.312 \\
\hline Italia ................................ & & & & 0 & & & & & & & 0 \\
\hline Japón ................................... & & & 7.955 .679 & 13.599 & & 347 & 10.695 & & & & 7.980 .320 \\
\hline Jordania ............................... & & 528 & & & & & & & & & 528 \\
\hline Kuwait ................................. & & 420 & 91.199 & & & & & & & & 91.619 \\
\hline Malasia ............................. & & 2.180 .324 & & & & 276.548 & & & & & 2.456 .872 \\
\hline 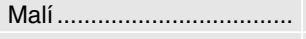 & & 16.740 & & & & & & & & & 16.740 \\
\hline Marruecos........................... & 11.969 & 204.509 & & & & & & & & & 216.478 \\
\hline México …......................... & & & 78.096 & & & 16.320 & & & & & 94.416 \\
\hline Nicaragua ........................ & & 51 & & & & & & & & & 51 \\
\hline Nigeria .............................. & & 148.294 & & & & & & & & & 148.294 \\
\hline Noruega ............................... & & 57.600 & & & & & & & & & 57.600 \\
\hline Nueva Zelanda .................. & & 19 & 0 & & & & & & & & 19 \\
\hline Omán ................................... & & 1.331 .112 & & & & & & & & & 1.331 .112 \\
\hline Panamá .............................. & & 15.248 & & & & & & & & & 15.248 \\
\hline Paraguay ............................. & & 105.788 & 480 & & & & & & & & 106.268 \\
\hline 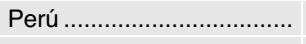 & 580 & 1.210 & & & & 893 & & & & & 2.683 \\
\hline Reino Unido ........................ & & & & & & & & & & 188.324 & 188.324 \\
\hline R.P. China ........................... & 287.450 & 1.748 .338 & 5.490 .496 & 4.648 .706 & & 2.329 & 4.533 & & & 130.150 & 12.312 .002 \\
\hline Rusia................................... & & & 2.352 .825 & 2.577 .939 & & 61.200 & & & & & 4.991 .964 \\
\hline Senegal.............................. & & 744 & & & & & 11.027 & & & & 11.771 \\
\hline Serbia ................................. & & 52.081 & 1.460 .434 & & & & & & & & 1.512 .515 \\
\hline Singapur ............................ & & & & & & & & & & 1.985 & 1.985 \\
\hline Sudafrica & & 390.806 & & & & & & & & & 390.806 \\
\hline Sudán del Sur.................... & & & & & & 2.981 & & & & & 2.981 \\
\hline Suiza.................................... & & 11.491 & 613.164 & & & 116 & 3.750 & & & 11.504 & 640.025 \\
\hline Tailandia.............................. & & 7.479 & 20.718 & & & & & & & & 28.197 \\
\hline Taiwán.................................... & & 3.738 & 4.148 & 427.232 & & 3.909 & & & & & 439.027 \\
\hline Turquía............................... & & 129.420 & & & & & & & & & 129.420 \\
\hline Uruguay .............................. & & 54.541 & & & & & & & & & 54.541 \\
\hline Venezuela ............................. & & & 2.760 & & & & & & & & 2.760 \\
\hline 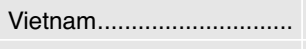 & & 793 & 24.518 & & & & & & & & 25.311 \\
\hline TOTAL & 2.559.427 & 12.958 .413 & 52.235 .788 & 9.704 .448 & 32.925 & 2.171.519 & 61.210 & 130.587 & & 1.364 .853 & 81.219 .170 \\
\hline
\end{tabular}


EXPORTACIONES ESPAÑOLAS DE MATERIAL DE DEFENSA...

\begin{tabular}{|c|c|c|c|}
\hline \multicolumn{4}{|c|}{$\begin{array}{l}\text { 26. EXPORTACIONES REALIZADAS DE PRODUCTOS Y TECNOLOGÍAS DE DOBLE USO CON INDICACIÓN } \\
\text { DE LA NATURALEZA DEL USUARIO FINAL, USO FINAL Y PORCENTAJE } \\
\text { PRIMER SEMESTRE } 2015\end{array}$} \\
\hline País & E. Privada & E. Pública & FFAA \\
\hline Andorra & 6,59 & 93,41 & \\
\hline Arabia Saudí ...... жан & 18,41 & 63,48 & 18,11 \\
\hline Argelia. & 100,00 & & \\
\hline Argentina & 34,57 & 65,43 & \\
\hline 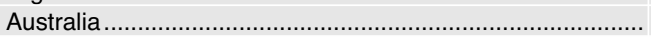 & 100,00 & & \\
\hline Bangladesh & 100,00 & & \\
\hline 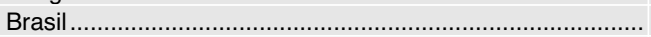 & 100,00 & & \\
\hline Canadá & 100,00 & & \\
\hline 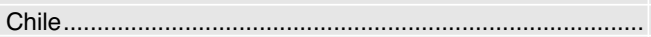 & 100,00 & & \\
\hline Colombia & 98,01 & 1,99 & \\
\hline 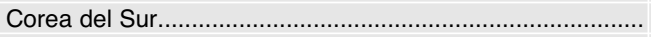 & 2,33 & 97,67 & \\
\hline Ecuador. & 100,00 & & \\
\hline Amiratos Árabes Unidos & 98,71 & 1,29 & \\
\hline Estados Unidos & 100,00 & & \\
\hline 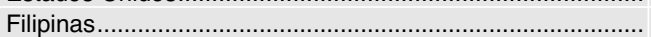 & 100,00 & & \\
\hline Francia & 100,00 & & \\
\hline Hong Kong (R.P. China) …………… & 100,00 & & \\
\hline 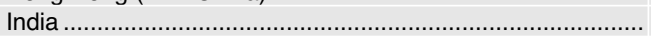 & 43,11 & 56,89 & \\
\hline Irak & 100,00 & & \\
\hline Irán & 21,38 & 78,62 & \\
\hline Israel .11 & 100,00 & & \\
\hline 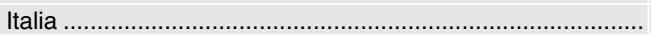 & 100,00 & & \\
\hline Japón & 100,00 & & \\
\hline Jordania & 100,00 & & \\
\hline Kuwait & 100,00 & & \\
\hline Malasia & 100,00 & & \\
\hline 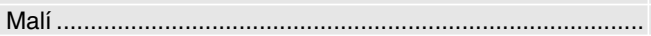 & 100,00 & & \\
\hline 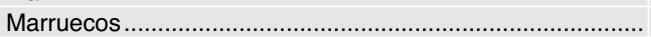 & 94,47 & 5,53 & \\
\hline México & 100,00 & & \\
\hline Nicaragua & 100,00 & & \\
\hline 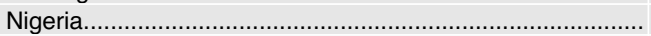 & 100,00 & & \\
\hline Noruega & 100,00 & & \\
\hline 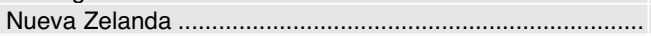 & 100,00 & & \\
\hline Omán & 100,00 & & \\
\hline Panamá. & 100,00 & & \\
\hline Paraguay & 100,00 & & \\
\hline Perú & 100,00 & & \\
\hline 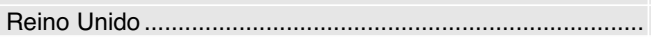 & 100,00 & & \\
\hline R.P. China & 99,92 & 0,08 & \\
\hline 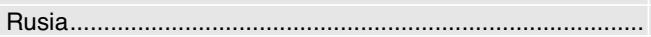 & 21,54 & 78,46 & \\
\hline Senegal. & 100,00 & & \\
\hline Serbia. & 100,00 & & \\
\hline Singapur & 100,00 & & \\
\hline Sudáfrica & 100,00 & & \\
\hline Sudán del Sur & & 100,00 & \\
\hline Suiza & 100,00 & & \\
\hline Tailandia & 84,32 & 15,68 & \\
\hline Taiwán & 2,69 & 97,31 & \\
\hline 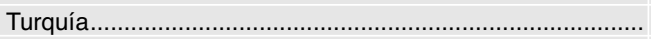 & 100,00 & & \\
\hline 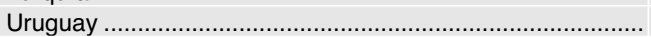 & 100,00 & & \\
\hline 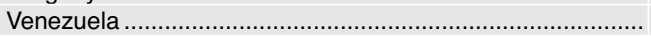 & 100,00 & & \\
\hline Vietnam & 100,00 & & \\
\hline
\end{tabular}

\begin{tabular}{|c|c|c|c|c|}
\hline \multicolumn{4}{|c|}{ 27. DENEGACIONES APLICADAS EN LA EXPORTACIÓN DE PRODUCTOS Y TECNOLOGÍAS DE DOBLE USO } \\
PRIMER SEMESTRE 2015
\end{tabular}




\section{Subdirección General de Comercio Internacional de Material de Defensa y Doble Uso}

\section{APLICACIÓN DE LA CLÁUSULA CATCH-ALL EN EXPORTACIÓN DE PRODUCTOS Y TECNOLOGÍAS DE DOBLE USO}

PRIMER SEMESTRE 2015

\begin{tabular}{|c|c|c|c|c|}
\hline País & Número & Producto & Motivo \\
\hline Irán............................... & 1 & Repuestos de válvulas de seguridad & $\begin{array}{l}\text { Situación de inestabilidad interna y riesgo de desvío a programas de } \\
\text { proliferación }\end{array}$ \\
\hline
\end{tabular}

Fuente: Subdirección General de Comercio Internacional de Material de Defensa y Doble Uso (Ministerio de Economía y Competitividad).

\begin{tabular}{|c|c|c|}
\hline \multicolumn{3}{|c|}{$\begin{array}{l}\text { 29. EXPORTACIONES REALIZADAS DE PRODUCTOS Y TECNOLOGÍAS DE DOBLE USO } \\
\text { SUPERIORES A } 10 \text { MILLONES DE EUROS } \\
\text { PRIMER SEMESTRE } 2015\end{array}$} \\
\hline País & Producto & Valor euros \\
\hline Estados Unidos .................... & Máquinas fresadoras, encintadoras y de mecanizado & 13.560 .640 \\
\hline TOTAL $\ldots \ldots \ldots \ldots \ldots \ldots \ldots \ldots \ldots \ldots \ldots$ & (1) & 13.560 .640 \\
\hline
\end{tabular}

\begin{tabular}{|c|c|c|}
\hline Categoría & Descripción 10 categorías & Relación de productos incluidos \\
\hline 0 & Materiales, instalaciones y equipos nucleares & $\begin{array}{l}\text { Reactores nucleares, plantas para la separación de isótopos de uranio natural, ura- } \\
\text { nio empobrecido y materiales fisionables, centrifugadoras de gas, espectrómetros } \\
\text { de masas y electrodos de grafito }\end{array}$ \\
\hline 1 & $\begin{array}{l}\text { Materiales, sustancias químicas «microorganismos» y «toxi- } \\
\text { nas» }\end{array}$ & $\begin{array}{l}\text { Equipos de protección y detección: trajes, guantes y calzado, dosímetros persona- } \\
\text { les, preimpregnados, herramientas, troqueles, moldes, mezcladoras continuas, má- } \\
\text { quinas para el devanado de filamentos, fluidos y sustancias lubricantes, fluoruros, } \\
\text { sulfuros, cianuros y derivados halogenados }\end{array}$ \\
\hline 2 & Tratamiento de los materiales & $\begin{array}{l}\text { Rodamientos, crisoles, máquinas-herramienta, prensas isostáticas, instrumentos } \\
\text { de medida de desplazamiento lineal y angular, robots, simuladores de movimien- } \\
\text { tos o mesas de velocidad y centros de mecanizado }\end{array}$ \\
\hline 3 & Electrónica & $\begin{array}{l}\text { Componentes electrónicos, circuitos integrados, microcircuitos de microprocesa- } \\
\text { dor, conjuntos de puertas programables, componentes de microondas, mezclado- } \\
\text { res y convertidores de frecuencia o generadores y detonadores explosivos accio- } \\
\text { nados eléctricamente }\end{array}$ \\
\hline 4 & Ordenadores & $\begin{array}{l}\text { Ordenadores electrónicos, híbridos, digitales, analógicos, de conjunto sistólico, neu- } \\
\text { ronales y ópticos }\end{array}$ \\
\hline 5 & Telecomunicaciones y «seguridad de la información» & $\begin{array}{l}\text { Equipos y sistemas de transmisión para telecomunicaciones, sistemas de comuni- } \\
\text { caciones subacuáticos, equipos de radio, cables de fibra óptica, equipos de tele- } \\
\text { medida y telecontrol y sistemas de seguridad de la información }\end{array}$ \\
\hline 6 & Sensores y láseres & $\begin{array}{l}\text { Acústica, tubos intensificadores de imagen, sensores ópticos, cámaras de instru- } \\
\text { mentos, óptica, láseres, gravímetros, sónares, hidrófonos y gradiómetros de gra- } \\
\text { vedad y sistemas de radar }\end{array}$ \\
\hline 7 & Navegación y aviónica & $\begin{array}{l}\text { Acelerómetros, giroscopios, GNSS, GPS y GLONASS, seguidores de estrellas, al- } \\
\text { tímetros, sistemas de control de vuelo hidráulicos, mecánicos, electroópticos y elec- } \\
\text { tromecánicos incluidos los de control por señales eléctricas (fly by wire)) }\end{array}$ \\
\hline 8 & Marina & $\begin{array}{l}\text { Vehículos sumergibles tripulados o no, y buques de superficie, hidroplanos, siste- } \\
\text { mas de visión subacuática, aparatos de buceo y natación subacuática. }\end{array}$ \\
\hline 9 & $\begin{array}{l}\text { Sistemas de propulsión, vehículos espaciales } \\
\text { y equipos relacionados }\end{array}$ & $\begin{array}{l}\text { Motores aeronáuticos o marinos de turbina de gas, lanzaderas espaciales y vehí- } \\
\text { culos espaciales, sistemas de propulsión de cohetes de propulsante sólido o líqui- } \\
\text { do, motores estatorreactores, turborreactores y turbofanes, cohetes de sondeo, mo- } \\
\text { tores híbridos para cohetes, equipos de apoyo al lanzamiento, cámaras ambientales } \\
\text { y anecoicas y vehículos de reentrada lanzamiento, cámaras ambientales y anecoicas } \\
\text { y vehículos de reentrada }\end{array}$ \\
\hline
\end{tabular}

Fuente: Subdirección General de Comercio Internacional de Material de Defensa y Doble Uso (Ministerio de Economía y Competitividad).

\begin{tabular}{|c|c|c|c|c|}
\hline \multicolumn{3}{|c|}{ 31. EVOLUCIÓN DE LAS EXPORTACIONES DE MATERIAL DE DEFENSA, DE OTRO MATERIAL Y DE PRODUCTOS } \\
Y TECNOLOGIAS DE DOBLE USO \\
PRMER SEMESTRE 2015
\end{tabular}

Fuente: Subdirección General de Comercio Internacional de Material de Defensa y Doble Uso (Ministerio de Economía y Competitividad). 